\title{
AN OVERVIEW OF BODY ARMOR AND SINGLE PLATE IMPACT DYNAMICS
}

\author{
A Thesis \\ presented to \\ the Faculty of California Polytechnic State University, \\ San Luis Obispo
}

\author{
In Partial Fulfillment \\ of the Requirements for the Degree \\ Master of Science in Mechanical Engineering
}

by

Thomas R. Matzinger

March 2017 
(C) 2017

Thomas R. Matzinger

ALL RIGHTS RESERVED 
COMMITTEE MEMBERSHIP

TITLE: An Overview of Body Armor and Single Plate Impact Dynamics

AUTHOR: Thomas R. Matzinger

DATE SUBMITTED: March 2017

COMMITTEE CHAIR: Tom Mase, Ph.D.

Professor of Mechanical Engineering

COMMITTEE MEMBER: Joseph Mellow, Ph.D.

Professor of Mechanical Engineering

COMMITTEE MEMBER: Jim Meagher, Ph.D.

Professor of Mechanical Engineering 


\begin{abstract}
An Overview of Body Armor and Single Plate Impact Dynamics
\end{abstract}

Thomas R. Matzinger

In the past, personal body armor was constructed of simple plates of highstrength alloys. However, with the advancement of modern combat and weaponry, particularly armor-piercing ammunition, personal body armor has evolved into more complex and effective metal, ceramic, and composite structures. This paper lays the groundwork for experimental and modeling methods used to understand the effectiveness of new armor designs. Focusing on the first layer of modern body armor, the "High Impedance" layer. Experiments measuring the change in velocity of bullets passing through aluminum and titanium plates were conducted. These experiments were then replicated through FEA simulation. 


\section{TABLE OF CONTENTS}

Page

LIST OF TABLES . . . . . . . . . . . . . . . . . . . . . . . . . vii

LIST OF FIGURES . . . . . . . . . . . . . . . . . . . . . . . . . viii

CHAPTER

1 INTRODUCTION . . . . . . . . . . . . . . . . . . . 1

1.1 An Overview of Modern Armor $\ldots \ldots \ldots \ldots$

1.2 Current Armor Designs . . . . . . . . . . . . . . . . 2

1.2.1 High-Impedance Layer . . . . . . . . . . . . . . . . 2

1.2.2 Energy Absorption Layer . . . . . . . . . . . . . . . 3

1.2.3 Protrusion Protection . . . . . . . . . . . . . . 3

2 EXPERIMENTAL DESIGN . . . . . . . . . . . . . . . . . . 5

2.1 Experimental Overview $\ldots \ldots \ldots \ldots \ldots \ldots$

2.2 Implementation and Limitations . . . . . . . . . . . . . 7

2.2.1 Target Plate . . . . . . . . . . . . . . . . . . . 7

2.2.2 Equipment and Setup . . . . . . . . . . . . . . . 7

3 SIMULATION . . . . . . . . . . . . . . . . . . . . 11

3.1 Model Overview . . . . . . . . . . . . . 11

3.2 Model Formulation . . . . . . . . . . . . . . . . . . . . 12

3.3 Meshing . . . . . . . . . . . . . . . . . . . . 14

3.3.1 Bullet Mesh . . . . . . . . . . . . . . . . . . . . . . 15

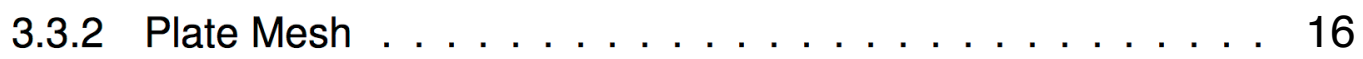

3.4 Material Model . . . . . . . . . . . . . . . . . . 19

3.4.1 Johnson and Cook Strength Model . . . . . . . . . . 20

3.4.2 Johnson and Cook Damage Model . . . . . . . . . . . . . 21

3.4.3 Material Properties . . . . . . . . . . . . . . . . . . . 22

4 RESULTS . . . . . . . . . . . . . . . . . . . . . . . . . . 25

4.1 Experimental Results . . . . . . . . . . . . . . . 25

4.1 .1 Chronograph Data . . . . . . . . . . . . . . 25 
4.1 .2 Photos of Experiment $\ldots \ldots \ldots 28$

4.1.3 Discussion/Observations of Experimental Results . . . . . 33

4.2 Modeling Results . . . . . . . . . . . . . . . . . . 34

4.2 .1 Velocity Results . . . . . . . . . . . . . . . . . 34

4.2.2 Conservation of Energy and Time step Selection . . . . . . 38

4.2 .3 Mesh Convergence . . . . . . . . . . . . . . . . . . 42

4.3 Experimental and Simulation Results . . . . . . . . . . . . 47

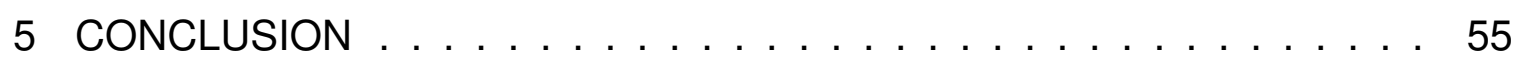

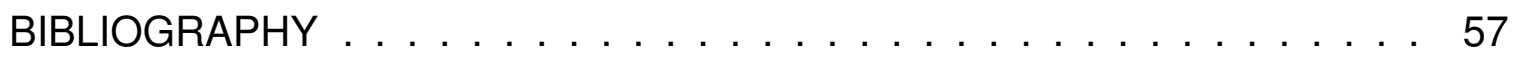




\section{LIST OF TABLES}

Table

Page

3.1 Material Properties generated by Matweb . . . . . . . . . 23

3.2 Johnson and Cook Material Model Properties [5] . . . . . . . . . 23

3.3 Johnson and Cook Material Damage Properties [5] . . . . . . . . . 24

4.1 M855 Exit Velocities $[\mathrm{m} / \mathrm{s}](2024$ aluminum) $\ldots \ldots \ldots \ldots \ldots$

$4.2 \quad$ M855 Exit Velocities $[\mathrm{m} / \mathrm{s}](\mathrm{Ti}-4-6) \ldots \ldots \ldots \ldots$

$4.3 \quad$ M855 Entrance Velocities $[\mathrm{m} / \mathrm{s}](\mathrm{Ti}-4-6) \ldots \ldots \ldots \ldots \ldots \ldots$

$4.4 \quad$ M855 Entrance Velocities [m/s] (Ti-4-6), statistics . . . . . . . . 27

4.5 Total Deformable Element Count for Various Levels of Mesh Refinement . . . . . . . . . . . . . . . . . . . . 35

4.6 Simulated Fully Deformable Exit Velocities in $\mathrm{m} / \mathrm{s}$, With Varying Element Count . . . . . . . . . . . . . . . . . . . . . 35

4.7 Simulated Rigid Core Exit Velocities in $\mathrm{m} / \mathrm{s}$, With Varying Element Count . . . . . . . . . . . . . . . . . . . . . 36

4.8 Percent change in energy balance for rigid body simulations, With Varying Element Count . . . . . . . . . . . . . . . . . . . 41

4.9 Percent change in energy balance for non rigid body simulations, With Varying Element Count . . . . . . . . . . . . . . . . . . 41 


\section{LIST OF FIGURES}

Figure

Page

$1.1 \quad$ Modern body armor schematic. . . . . . . . . . . . . 2

$2.1 \quad$ Idealized experimental setup $\ldots \ldots \ldots \ldots \ldots$

2.2 Diagram of San Luis Obispo Sheriff's Department shooting range, and experimental setup. . . . . . . . . . . . . . 8

2.3 Full experimental setup, including target plate and "Gamma Master Chrony" chronograph . . . . . . . . . . . . . . . . . 9

3.1 Example of a trapped and overly elongated element. . . . . . . 13

3.2 High density cubic M855 mesh, of bullet jacket (blue), steel core (yellow), and lead penetrator (purple). . . . . . . . . . . . . 14

3.3 High density linear M855 mesh, of bullet jacket (blue), steel core (yellow), and lead penetrator (purple). . . . . . . . . . . . 15

3.4 Cross section of bullet interior, showing a classic example of the butterfly mesh technique. . . . . . . . . . . . . . . 15

3.5 Bullet tip region of high density mesh highlighting both a 2:1 mesh transitions, and three dimensional butterfly mesh. . . . . . . . . 16

3.6 High density target plate mesh, illustrating the partition of the plate into the "rigid body" and "impact" zones.

3.7 High density target plate mesh, illustration the $3: 1$ transition between "rigid body" and "impact" zones. . . . . . . . . . . . . . . 18

4.1 Entrance holes in an aluminum (left) and titanium (right) 0.032" thick plates. . . . . . . . . . . . . . . . . . . 28

4.2 Entrance holes in an aluminum (left) and titanium (right) 0.063 " thick plates. . . . . . . . . . . . . . . . . . . . . 29

4.3 Exit holes in an aluminum (left) and titanium (right) 0.032 " thick plates. . . . . . . . . . . . . . . . .

4.4 Exit holes in an aluminum (left) and titanium (right) 0.063 " thick plates. . . . . . . . . . . . . . . . . . . 30

4.5 Entrance holes in an aluminum (left) and titanium (right) 0.125 " thick plates. . . . . . . . . . . . . . . . . . . . . 
4.6 Exit holes in an aluminum (left) and titanium (right) 0.125 " thick plates. . . . . . . . . . . . . . . . . . . . 31

$4.7 \quad 0.032$ " titanium plate displaying brittle material petaling behavior. . 31

4.8 M855 shrapnel spread pattern created after passing through 0.063 " aluminum plate. . . . . . . . . . . . . . . . 32

4.9 M855 shrapnel spread pattern created after passing through 0.063 " titanium plate. . . . . . . . . . . . . . . . 32

4.10 Part velocities for simulation of impact with 0.032 " aluminum plate, in which bullet core and lead were modeled as rigid. . . . . . . . . 36

4.11 Part velocities for simulation of impact with 0.032 " aluminum plate, in which all components were modeled as fully deformable. . . . .

4.12 Energy balance results for simulation of fully deformable impact with 0.25 " aluminum plate. . . . . . . . . . . . . . . . .

4.13 Time step results for simulation of fully deformable impact with 0.25 " aluminum plate. . . . . . . . . . . . . . . . . . . .

4.14 Convergence study of the effects of mesh density on 0.32 " plates plate impacts. . . . . . . . . . . . . . . . .

4.15 Convergence study of the effects of mesh density on 0.63 " plates plate impacts. . . . . . . . . . . . . . . . . . . .

4.16 Convergence study of the effects of mesh density on 0.125 " plates plate impacts. . . . . . . . . . . . . . . . .

4.17 Comparison of experimental and simulated Results of aluminum plates . . . . . . . . . . . . . . . . .

4.18 Comparison of experimental and simulated Results of titanium plates . . . . . . . . . . . . . . . . .

4.19 Simulated impact of an M855 bullet with 0.032" thick aluminum plate, represented with a high density mesh. . . . . . . . . . .

4.20 Simulated impact of an M855 bullet with 0.063 " thick aluminum plate, represented with a high density mesh. . . . . . . . . . . .

4.21 Simulated impact of an M855 bullet with 0.125 " thick aluminum plate, represented with a high density mesh. . . . . . . . . . 52

4.22 Simulated impact of an M855 bullet with 0.250 " thick aluminum plate, represented with a high density mesh. . . . . . . . . . 53

4.23 Side view of a simulated impact of an M855 bullet with 0.250 " thick aluminum plate, represented with a high density mesh. . . . . . .

4.24 Three quarters view of a simulated impact of an M855 bullet with 0.032 " thick aluminum plate, represented with a high density mesh. 54 


\section{CHAPTER 1}

\section{INTRODUCTION}

\subsection{An Overview of Modern Armor}

In the past, personal body armor was constructed of simple plates of high-strength alloys. However, with the advancement of modern combat and weaponry, particularly armor-piercing ammunition, personal body armor has evolved into more complex and effective metal, ceramic, and composite structures. Soldiers require a personal body armor solution light enough to be worn comfortably with a full load and packed multiple days through rough terrain. Additionally this armor must be able to withstand multiple impacts, ideally from small arms up to high-power munitions.

The current trend is towards the use of technical ceramics (aluminum oxide, silicon carbide, aluminum nitrates, etc.) in combination with polymers such as paraaramid synthetic fibers (i.e. Kevlar) and ultra-high-molecular-weight polyethylene (UHMWPE, trade name Dyneema) [8]. The ceramics are used to fracture and deform the bullet on impact, while the synthetic fibers absorbs its now dispersed kinetic energy.

Ceramics present a high-strength and light-weight alternative to more durable materials, such as titanium. However, this comes at the cost of durability. Specifically, the tendency of ceramics to fracture makes them a less than ideal choice for armor 
sustaining repeated impacts - a likely scenario in the middle of combat [2]. Although briefly extremely effective, this comes at the cost of relatively high fragility for a material in combat environments. Because of these properties, ceramics have been a useful, but imperfect solution.

\subsection{Current Armor Designs}

Most modern armor consists of three main components as seen in Figure 1.1. Firstly, a high-impedance layer that fractures and deforms the bullet upon initial impact. Secondly, an energy dissipation layer designed to absorb as much of the bullet's kinetic energy as possible. Thirdly, a final protrusion protection layer meant to protect the wearer from any nominal velocity remaining in the bullet fragments. [8]

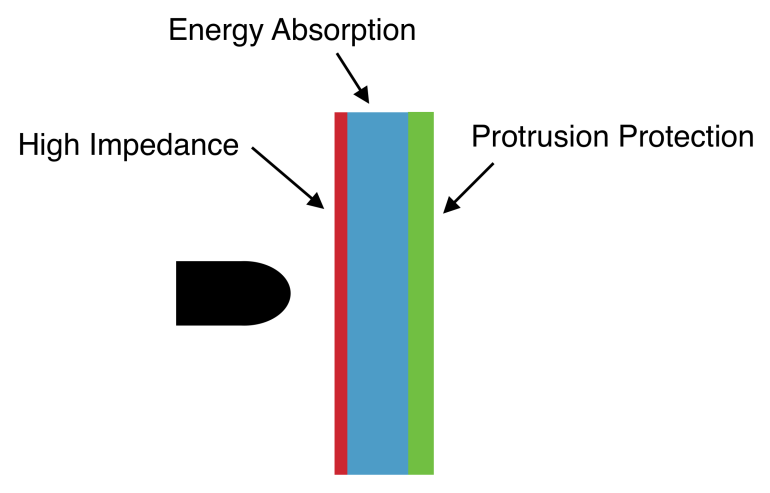

Figure 1.1 - Modern body armor schematic.

\subsubsection{High-Impedance Layer}

The high-impedance layer greatly determines the effectiveness of the energy absorption layer. By deforming and fracturing the bullet, the high-impedance layer causes energy to be dispersed over a greater area. Thus, the force of the impact is 
borne by a greater number of the woven composite fibers of the second layer [8].

Both harder and lighter weight than metal, ceramic is the popular modern choice for the initial layer of composite armor [4]. However, ceramics pose their own set of challenges. When a ceramic is penetrated the resulting fractures are rarely localized. Additionally, the brittle nature of ceramics make fractures more likely to occur in day-to-day scenarios. A main focus of this paper was to consider the viability of an alternative material choice for the high-impedance layer, specifically Ti-6Al-4V (Grade 5). Grade 5 is considered to be the best alloy available today for ballistics due to its combination of high strength and light weight characteristics [7].

\subsubsection{Energy Absorption Layer}

The second layer is intended to absorb and dissipate the majority of the bullets' kinetic energy. In addition to simply absorbing energy, truly optimized armors must attempt to dissipate the shock wave caused by impact. This dissipation occurs through the fibers of the composite, orthogonal to the impact. Thus, materials with high speeds of sound (high density) in the direction perpendicular to impact better distribute local material stresses, resulting in significant stress wave attenuation [8]. Some modern armors even go so far as to include layers specific to this task.

Materials most suited to this endeavor include kevlar fiber reinforced polymers (KFRPs), carbon fiber reinforced polymers (CFRPs), and ultra-high-molecular-weight polyethylene fabric composites (UHMWPE) [8]. These materials all offer a great deal of energy absorption through plastic deformation and material damage accumulation. 


\subsubsection{Protrusion Protection}

Unlike the energy absorption layer, the final layer is not meant to absorb energy through deformation. Instead, it is intended to catch any final fragments that pass through the first two layers with nominal velocity. Ideally this layer provides a backing for the energy absorption layer preventing delimitation of the composite[8]. A balance must be struck in this final layer so that it may deform with the second layer to better absorb energy, while at the same time not penetrating to far into the body. The malleability of most metal, and its homogenous structure, make it a good choice for this final layer. 


\section{CHAPTER 2}

\section{EXPERIMENTAL DESIGN}

\subsection{Experimental Overview}

The focus of this paper is impact upon the high-impedance layer only. Modern body armor has evolved into a complex structure of multiple materials with specific tasks modeling and study of the energy absorption and protrusion protection layers has been left for future work. In order to build an accurate model of the complete structure it is best to first understand its individual components.

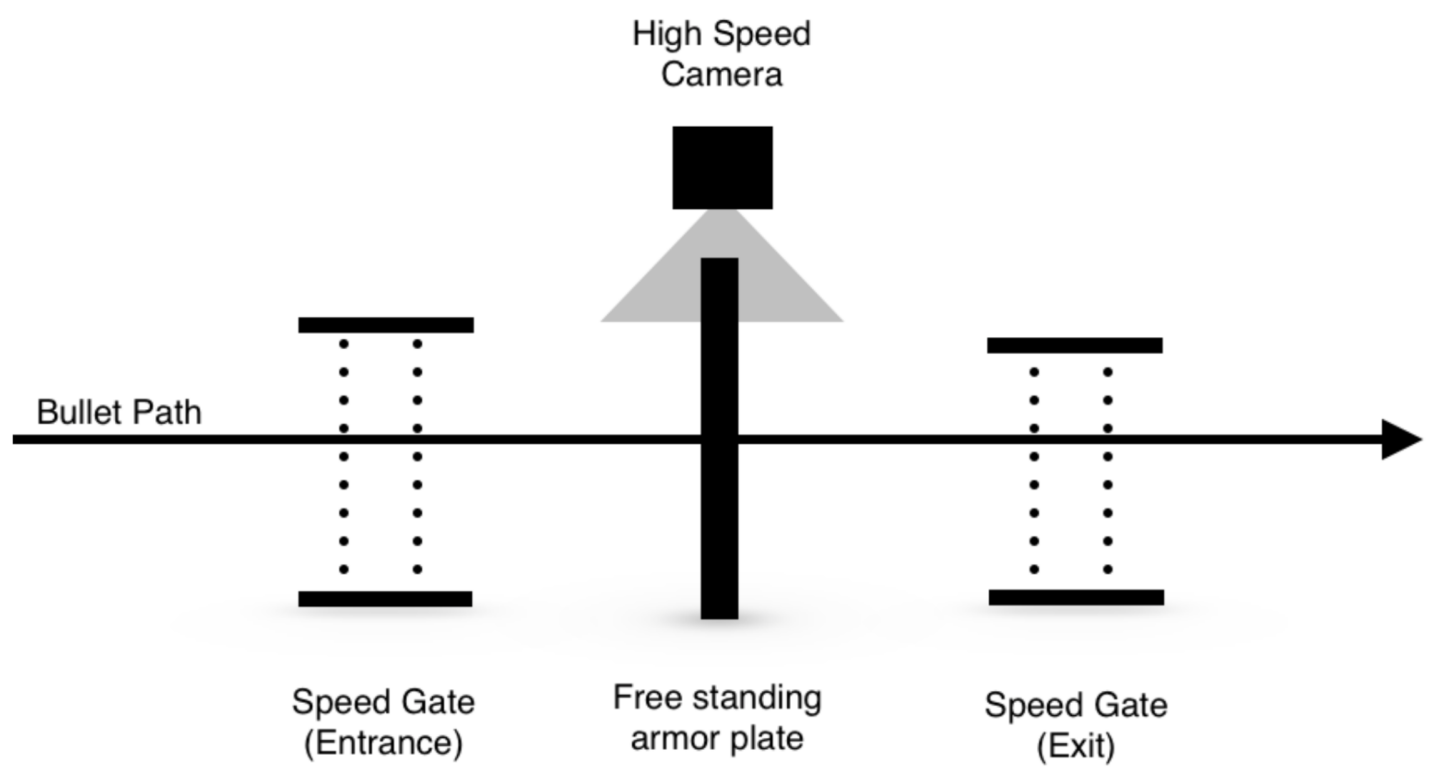

Figure 2.1 - Idealized experimental setup

Quantifying a high speed impact is difficult. In order to film the actual impact event in significant detail a camera capable of shooting 1,000,000 frames per sec- 
ond is required. In cases where the bullet does not pass through its target, the protrusion caused by the impact is measured $[3,4]$. However, our target plate is not designed to stop the $5.56 \times 45 \mathrm{~mm}$ NATO round used in testing so we must find another way to quantify the bullet's interaction with the target plate.

The basic concept of the experiment, conducted in this thesis, is simple. As shown in Figure 2.1, the experimental setup uses chronographs to measure both bullet entrance and exit velocity. The target plate itself is left unconstrained, and a high speed camera is used to film the impact. The purpose of the camera was not to see the actual impact event but to measure the speed of the plate after impact. As such, a more readily sourced 10,000 frame-per-second camera was used. With the plate and bullet measurements two good metrics of comparison between experiment and simulation have been established; namely, the unconstrained plate's velocity after impact, and the exit velocity of the bullet.

As seen in Figure 2.1, each trial of this experiment consisted of a single metal target plate. By varying the thickness of the plate, the aim was to establish a trend in the velocities of the bullet and plate, following their collision. This trend was observed as plate thickness is varied. By testing an increasing thickness of plate it is observed at what thickness the bullet begins to fracture; fragmentation and dispersal of kinetic energy being the ultimate goal of the high-impedance layer.

The round chosen to conduct these tests, the $5.56 \times 45 \mathrm{~mm}$ NATO round, is commonly referred to as the M855 green tip round. With a lead core, steel penetrator, and copper alloy jacket it does not meet the Bureau of Alcohol, Tobacco, Firearms and Explosives' technical definition of an armor piercing (AP) round, since it does not have a purely solid steel core. Though it is not considered to be a truly "armor 
piercing" round, M855s are one of the most commonly used types of ammunition on the modern battlefield, rendering all but the toughest body armor ineffective [2].

\subsection{Implementation and Limitations}

\subsubsection{Target Plate}

Choice of target materials was restricted by three main issues. Firstly, as all materials were purchased from McMaster-Carr ${ }^{1}$, so material size and selection was limited. Secondly, the availability of advanced material modeling parameters (Johnson Cook) was limited to those found online and in published papers. Finally, material cost was a limiting factor. As a result, only a limited number of trials could be attempted especially in the case of Ti-6Al-4V.

Given these restrictions, Ti-6Al-4V and aluminum 2024 were the final materials of choice. Sold in 6" $\times 6$ " plates, several thicknesses of each material were purchased. Specifically the plates were thicknesses of 0.032 ", 0.063 ", 0.125 " and 0.250 ". Ti-6Al-4V was chosen as its strength and weight makes it one of the best materials currently available for armor plating. Aluminum 2024 was chosen as a cheaper alternative to Ti-6Al-4V. Though it is unlikely to be a good choice for armor, grade 2024 is a relatively harder grade of aluminum and provides data useful in verifying our model.

\subsubsection{Equipment and Setup}

All experimental testing was conducted at the San Luis Obispo Sheriff's Department firing range on October $1 \mathrm{st}, 2015^{2}$. An AR-15 riffle, provided by the sheriff

\footnotetext{
${ }^{1}$ http://www.momaster.com

${ }^{2}$ Thank you to the San Luis Obispo Sheriff's Department, and Officer ${ }^{* * *}$ for facilitating access to the county firing range.
} 
department, was used in combination with M855 military surplus ammunition produced by Winchester. Figure 2.2 provides an aerial view of the test facility.

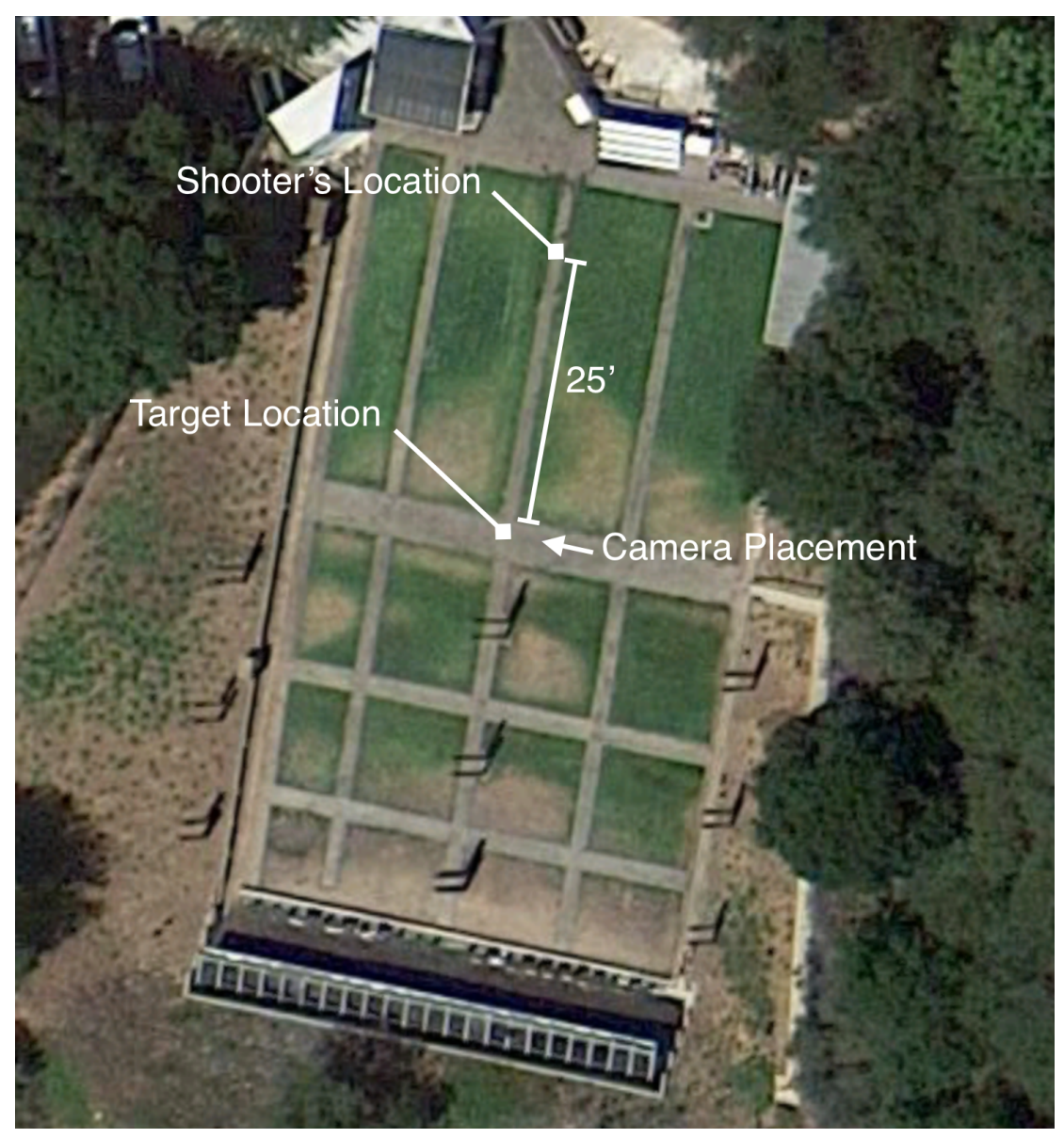

Figure 2.2 - Diagram of San Luis Obispo Sheriff's Department shooting range, and experimental setup.

A chronograph, the "Gamma Master Chrony", was used to measure the entrance and exit velocities of the bullet. It does this with two photo sensors separated by a known distance. It is capable of measuring velocity with an accuracy of $0.5 \%$ of its nominal value. Initially the chronograph was placed in front of the target plate, 25 feet from the shooter. Ten shot velocities were then recorded to establish entrance velocity, to a reasonable degree of certainty. The chronograph was then moved 
behind the supported target plate, and into the experimental configuration shown in Figure 2.3.

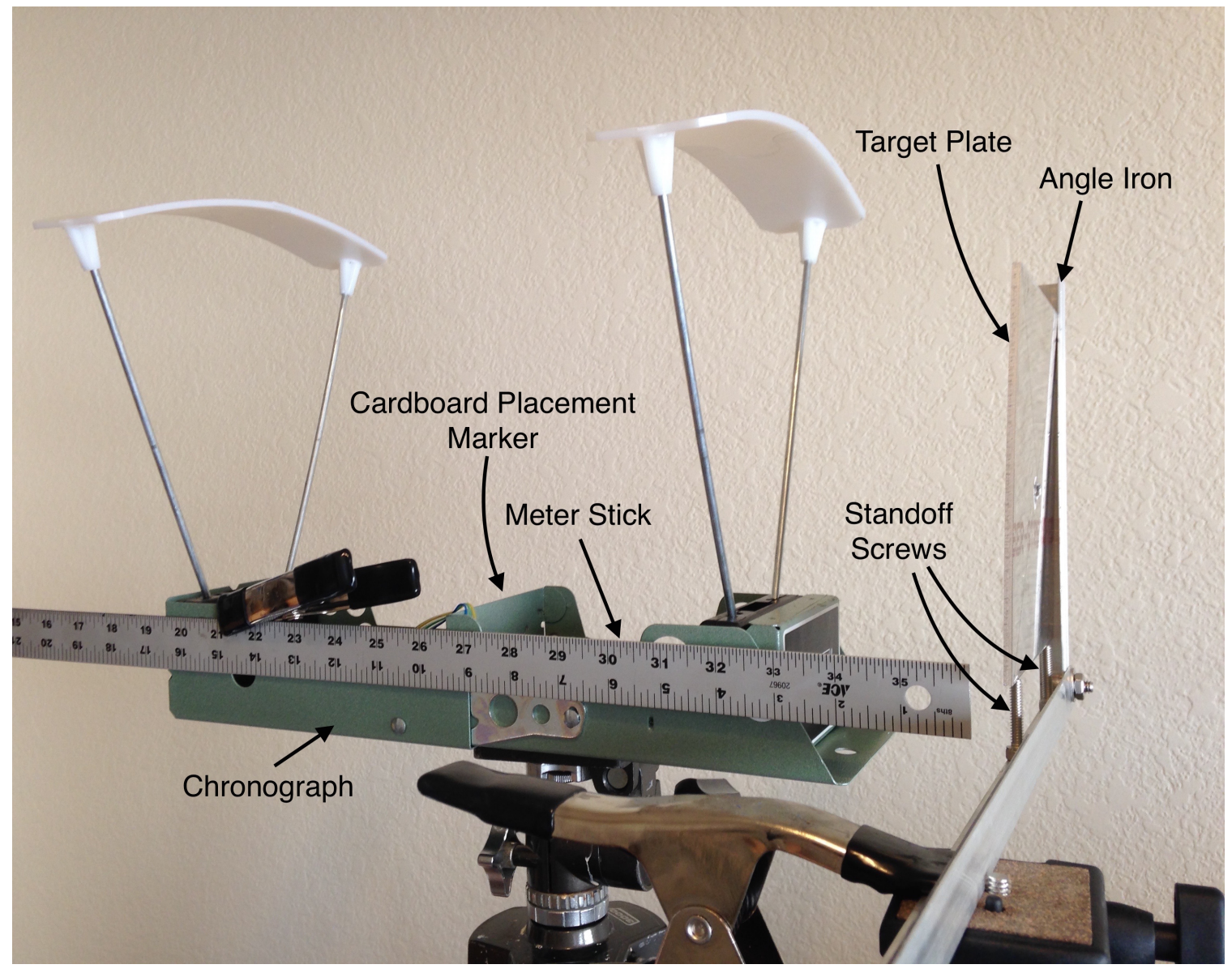

Figure 2.3 - Full experimental setup, including target plate and "Gamma Master Chrony" chronograph

The plate was positioned in front of the chronograph with aluminum angle attached to a tripod. In order to increase the accuracy of the velocity data gathered from the high speed camera, it was important to prevent rotation of the target plate. Thus, the target plate was balanced on two $3 / 4$ inch long socket head cap screws as seen in Figure 2.3. In this way any frictional force that would cause plate rotation was eliminated. 
In addition to the chronograph and high speed video, a more qualitative method was used to estimate fragmentation. Specifically sheets of cardboard were suspended between the two chronograph photo sensors as noted in Figure 2.3. Consequently, if the chronograph was unable to capture the velocity data of highly fragmented bullets, it would be possible to verify that fragmentation was in fact the issue. 


\title{
CHAPTER 3
}

\author{
SIMULATION
}

\subsection{Model Overview}

Impact with the high-impedance layer can result in two scenarios: one in which the bullet penetrates and passes through the plate with minimal impedance, or two, in which the bullet encounters the high-impedance layer and is significantly deformed and/or fractured. This second scenario is both the desired effect and the most difficult to model. In this second case both the plate and bullet undergo significant deformations, with the bullet oftentimes separating into multiple fragments. The described modeling problem deals with large deformations and crack propagation, and additionally due to the high speeds at impact material properties are also strain rate dependent.

These difficulties are not insurmountable. Initially, several simulation methods were considered including Lagrangian methods, Smoothed Particle Hydrodynamics (SPH), and Arbitrary Lagrangian Eulerian (ALE) formulations. It has been noted that, when used correctly, Lagrangian techniques hold up to, or outperform both SPH and ALE methods in testing with $9 \mathrm{~mm}$ rounds penetrating thin copper plates [1]. However, when encountering larger bullet deformations against thicker plates, ALE techniques may be considered for future work. 


\subsection{Model Formulation}

Lagrangian-based ballistic models often utilize one of several methods to overcome common obstacles discussed above. Specifically, the "the pilot hole" method, element erosion method, and node splitting. The first involves removing a small group of elements along the predetermined path of the bullet. The second simply removes (or erodes) highly damaged or deformed elements [1]. Neither method is physically representative, requiring unrealistic ad hoc knowledge of the problem at hand, so that erosion parameters can be tuned to match experimental data [7]. This obviously does not lend itself well to predictive modeling. A third option - node splitting - is physically representative but computationally intensive.

Initially, element formulation presented a significant challenge. The high speeds and large deformations simulated in this model require a high mesh density. This, combined with the computational requirements of node splitting, resulted in unworkably long runtimes on the available computers, contact issues, and negative volumes. Fortunately, the use of higher order elements, supported by IMPETUS significantly reduced these issues.

Through the use of cubic element formulations and GPU acceleration, relative runtimes were greatly reduced. In addition to eliminating many of the previously mentioned issues associated with linear elements, larger elements with more degrees of freedom have the added benefit of improving the computational times associated with node splitting. Especially since the IMPETUS solver architecture allows contact solutions to be done in parallel on the computer's GPU. These reductions in computational demands are realized at the cost of reduced fragmentation sites. Since only the corners nodes of the cubic element can break free, fragmentation 
now occurs over larger element regions, increasing the possibility of mesh dependance.

Even with the use of higher order elements and node splitting, difficulties arise. Certain elements often become "trapped" between the advancing front of the bullet, creating overly small element thicknesses and reducing simulation time step (Figure 3.1). As a result, despite all efforts, some form of element erosion is ultimately necessary.

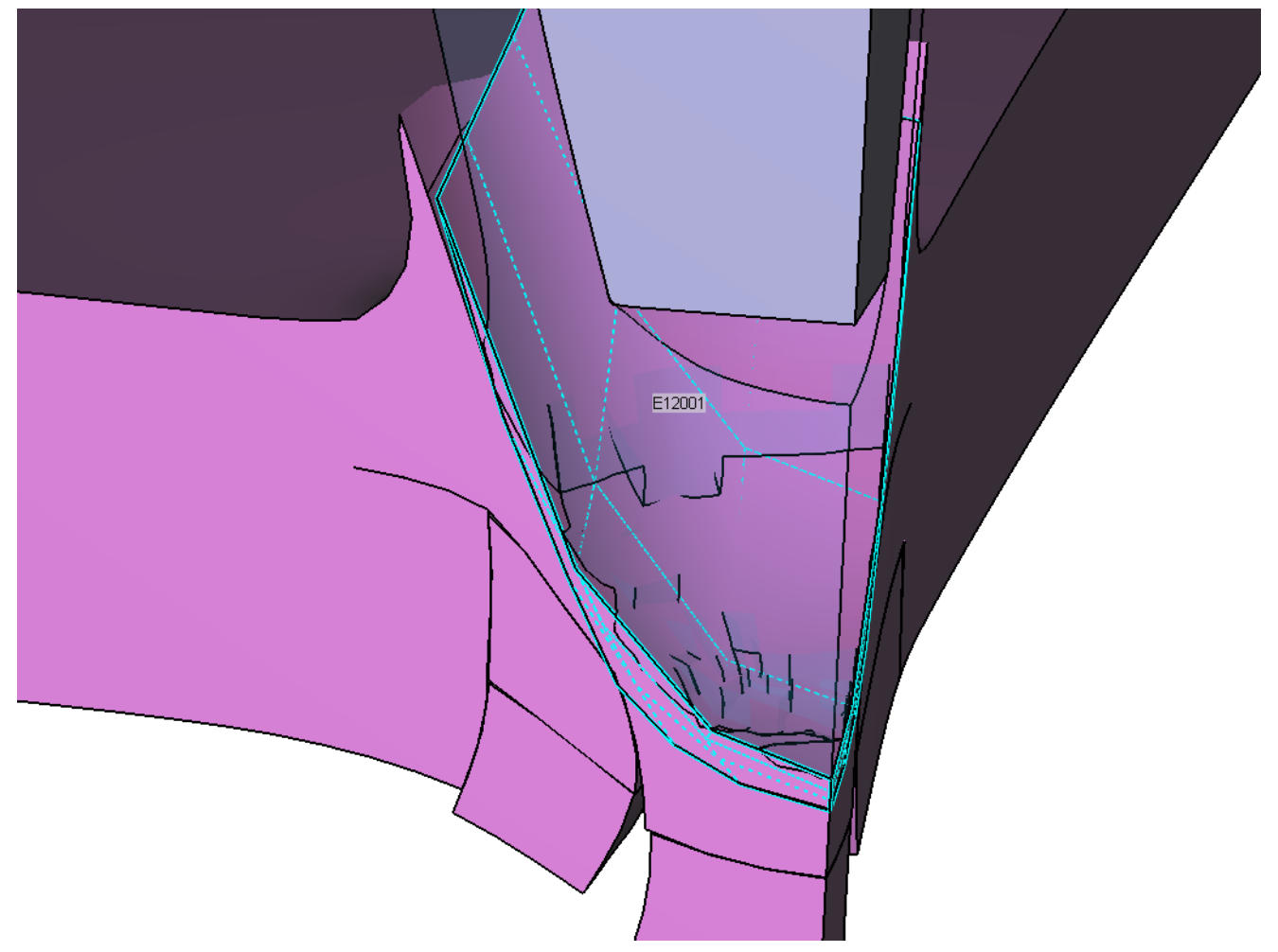

Figure 3.1 - Example of a trapped and overly elongated element.

In contrast to stress-based element erosion, time step based element erosion was 
used. So instead of eroding elements based on an arbitrary stress parameter, instead remove only the elements that have become so distorted as to be no longer functional. This makes the unrealistic phenomena of eroding elements a function of the computational resources available, instead of an arbitrary or experimental parameter. Additionally, since time step calculations are based, in part, on element thickness, erosion occurs mostly in elements that have been made too thin. This effect can be seen as similar to the actual phenomena of two objects splitting apart.

\subsection{Meshing}

A considerable amount of care was taken in developing highly regular meshes, across all mesh densities, for both the bullet and the plate. The use of the program TrueGrid allowed for easy parameterization of element counts, and allowed for the quality of the mesh to be measured. Meshes were initially generated in TrueGrid with simple linear hexahedral elements (Figure 3.3), and then interpolated into cubic elements using IMPETUS (Figure 3.2).

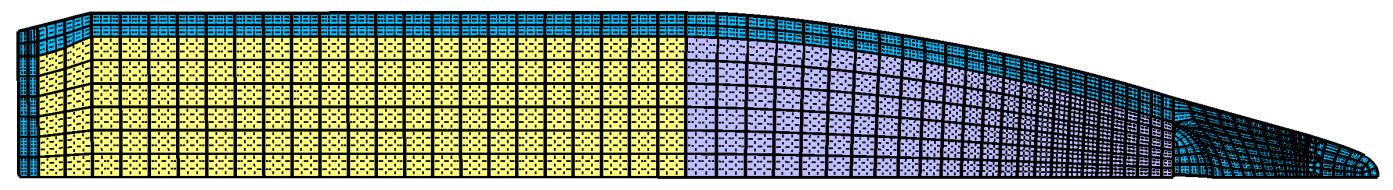

Figure 3.2 - High density cubic M855 mesh, of bullet jacket (blue), steel core (yellow), and lead penetrator (purple). 


\subsubsection{Bullet Mesh}

The M855 bullet mesh was constructed as three separate components: the bullet jacket, steel core, and lead penetrator. Each component consisting of at least one butterfly mesh, an example of which can be seen in Figure 3.4. The Butterfly mesh technique being the most common method to transition from a solid central core to a curved outer surface, while maintaining high mesh quality. In the highest density version of the mesh, Figure 3.3 , three main considerations were made.

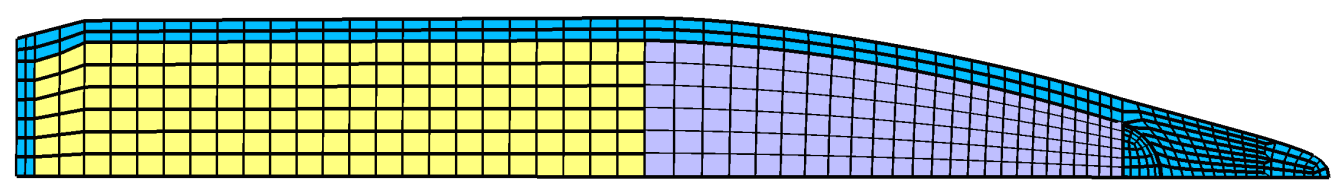

Figure 3.3 - High density linear M855 mesh, of bullet jacket (blue), steel core (yellow), and lead penetrator (purple).

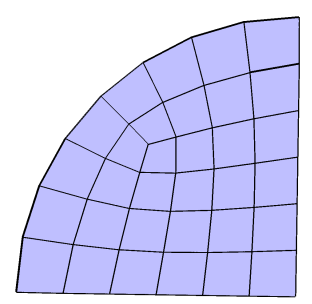

Figure 3.4 - Cross section of bullet interior, showing a classic example of the butterfly mesh technique.

Firstly, no element was allowed to have an aspect ratio greater than 3:1, excepting 
some areas directly next to the butterfly zone. Secondly, all surface regions were smoothed with a combination of TrueGrid's elliptical and absolute smoothing algorithms. Finally, all mesh regions in contact with one another were forced to have identical node positions. This helps the contact algorithm to prevent unwanted solid body intrusion. In order to prevent the size of the elements in the Bullet tip from dominating the mesh size of the rest of the bullet, a 2:1 element transition was used, as seen in Figure 3.5.

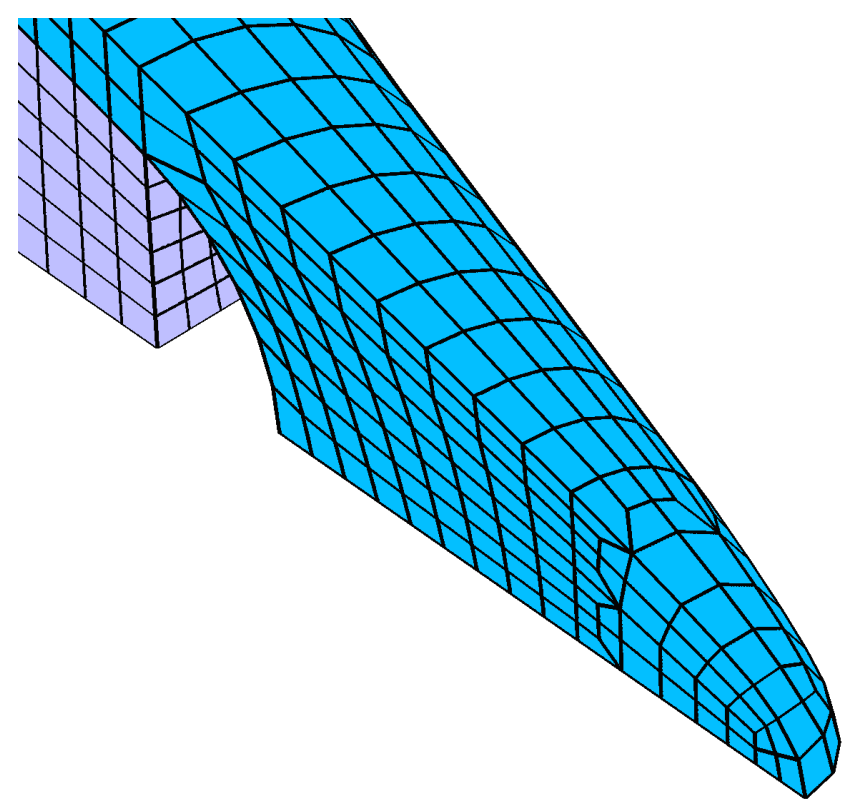

Figure 3.5 - Bullet tip region of high density mesh highlighting both a 2:1 mesh transitions, and three dimensional butterfly mesh.

\subsubsection{Plate Mesh}

In order to build a model most similar to the actual experiment, the entirety of the plate was modeled (Figure 3.6). Unfortunately, if done with equally sized elements, the mesh density near the zone of impact would be either be too sparse, or require impossibly long simulation runtimes due to high element counts. 


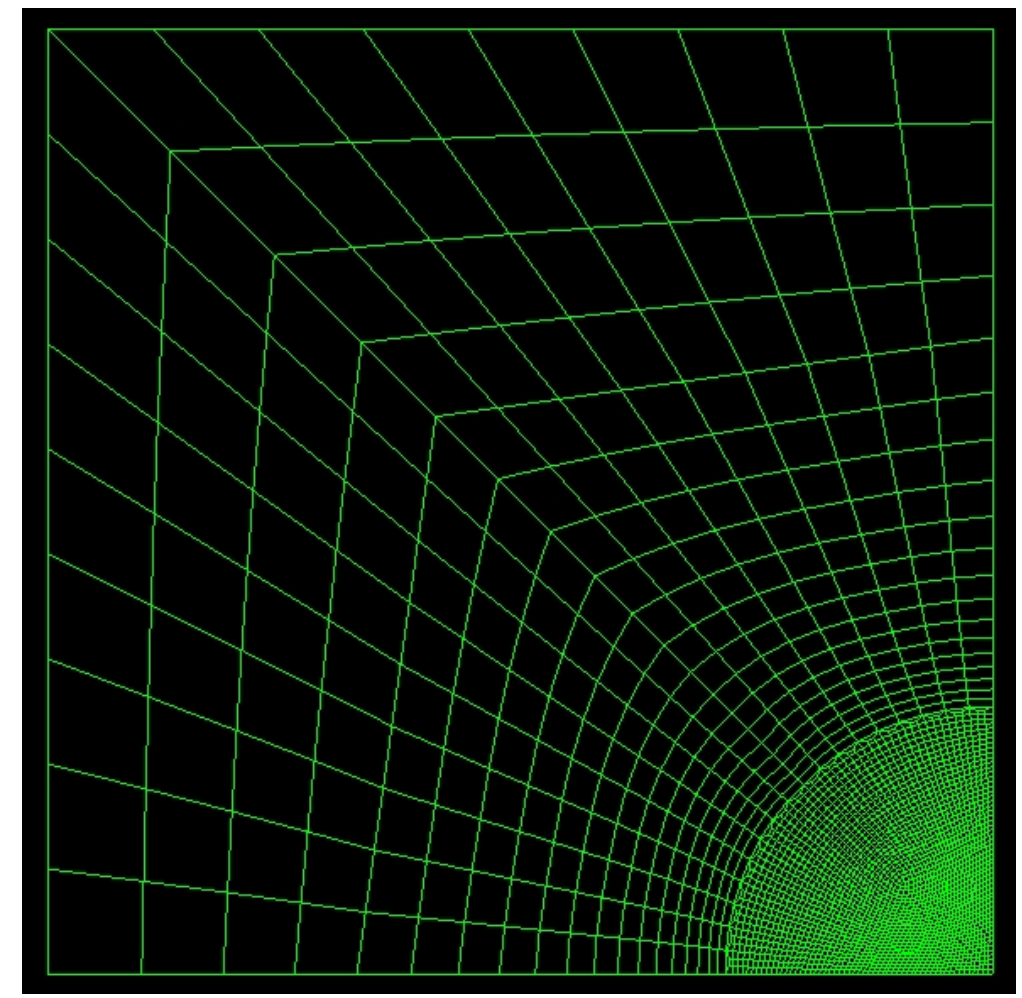

Figure 3.6 - High density target plate mesh, illustrating the partition of the plate into the "rigid body" and "impact" zones. 
Thus, the plate was split into two regions; the rigid body zone, which mainly supplied the required plate mass, and impact zone, which simulated the bullet impact. The division between the two was defined by an estimate of where the plates yield stress would reach approximately 50 percent.

The plate mesh in the impact zone was sized to maintain an aspect ratio below 1:3. The linear mesh was generated in TrueGrid, and the cubic mesh was generated in IMPETUS. Elliptical and absolute spacing algorithms were also used in order to improve element orthogonality. Additionally, a 3:1 (Figure 3.7) mesh transition was used between the impact and rigid body zones.

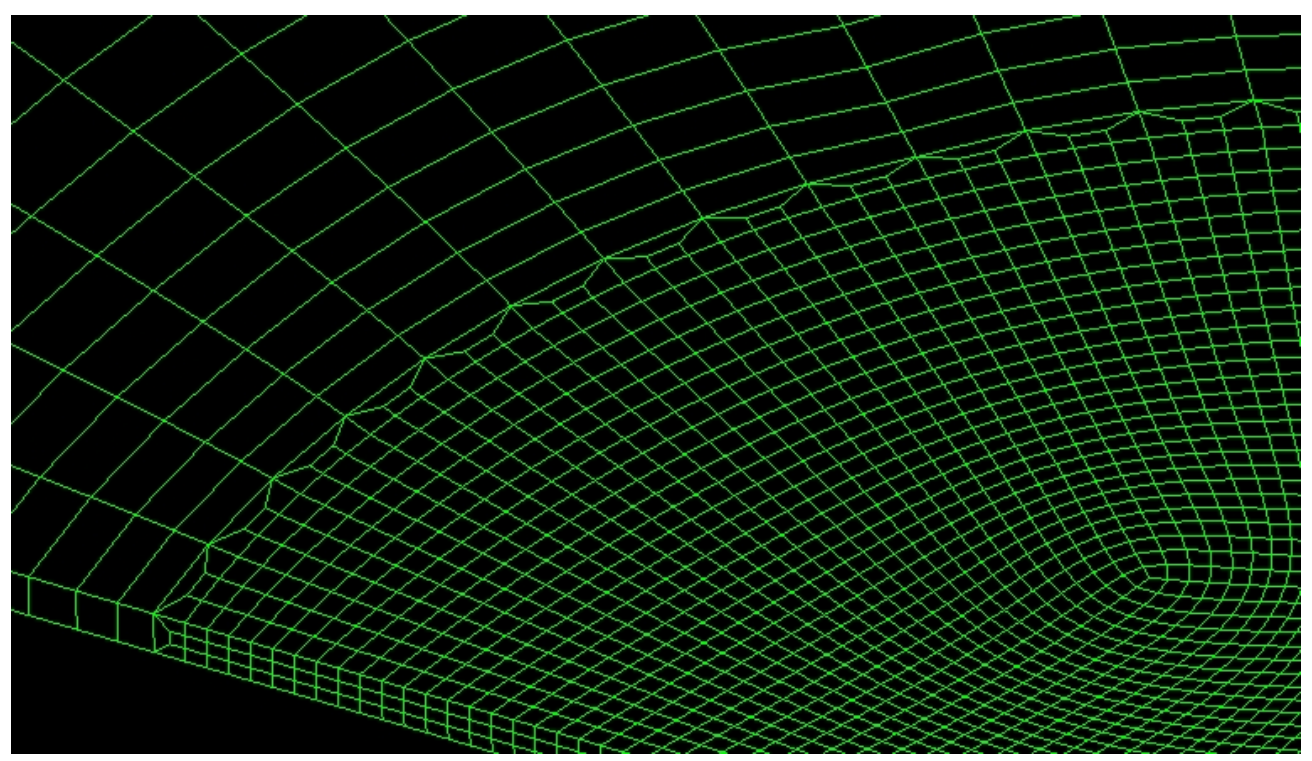

Figure 3.7 - High density target plate mesh, illustration the 3:1 transition between "rigid body" and "impact" zones.

Even with this greatly reduced area of high density mesh, computational limitations were encountered. Ideally the mesh geometry should have no impact on simulation results. However, as will be discussed later, in greater detail, with a sim- 
ple rectangular mesh the plate elements did not split as would be expected. That is to say "petaling" did not occur in the simulations. Thus, the plate mesh was generated using the "butterfly" technique. This resulted in a more radially distributed mesh, which resulted in nodes that split in a way more in line with experimental results.

\subsection{Material Model}

When considering ballistic impacts it is important to remember that standard linear material models are insufficient because they do not consider strain rate effects. Thus, a more comprehensive material model is required. Two such models were initially considered: the Johnson-Cook (JC) and the Zerilli-Armstrong (ZA) strength models. The JC model is a phenomenological model based purely on empirical tests such as Hopkinson Bar tests and cylinder impact tests, making material parameter calibration relatively easy [6]. The ZA model is based on simplified dislocation mechanics, with tailored equations for both face-centered cubic, and bodycentered cubic materials [7]. Because the form of the ZA model is mathematically based on the material's structure, some consider it to be a superior model [7]. However, the JC model was ultimately chosen. This is because it couples strain rate with temperature, an important consideration in ballistics testing. Additionally, material parameter testing is easier for the $\mathrm{JC}$ model, and consequently material parameters are widely available in the public domain. 


\subsubsection{Johnson and Cook Strength Model}

The Johnson and Cook strength model describes the behavior of a material after it has reached its yield point. That is, the three dimensional geometric surface that defines the stress-strain relationship of a material. This JC flow surface is defined by Equation (3.1) [10] . The three products seen in Equation (3.1) describe the effects of strain hardening, strain-rate effects, and thermal softening respectively [9].

$$
\sigma_{\text {yield }}=\left[A+B\left(\bar{\varepsilon}^{p}\right)^{n}\right]\left[1+C \ln \left(\dot{\varepsilon}^{*}\right)\right]\left[1+\left(T^{*}\right)^{m}\right]
$$

The first term, A, represents the initial yield strength of the material at room temperature (strain rate of $1 / \mathrm{s}$ ) and can be approximated as the quasi-static yield strength of the material [7]. Parameter A, along with density, specific heat, and melt temperature are the only material parameters associated with the model. The rest, B, $\mathrm{N}, \mathrm{C}$ and $\mathrm{M}$ are curve fitting parameters [9].

$$
\begin{aligned}
T^{*} & =\frac{T-T_{\text {room }}}{T_{\text {melt }}-T_{\text {room }}} \\
\bar{\varepsilon}^{p} & =\int_{0}^{t} d \bar{\varepsilon}^{p} \\
\dot{\varepsilon}^{*} & =\frac{\bar{\varepsilon}^{p}}{\dot{\varepsilon}^{0}}
\end{aligned}
$$

Within the JC flow surface (3.1) we find the additional terms $T^{*}, \bar{\varepsilon}^{p}$, and $\dot{\varepsilon}^{*}$. Where $T^{*}$ describes the normalized temperature as seen in Equation (3.2). Effective plastic strain, $\bar{\varepsilon}^{p}$, is defined in Equation (3.3). And $\dot{\varepsilon}^{*}$ describes the the non-dimensional ratio of the effective plastic strain to the reference strain rate (commonly $1 / \mathrm{s}$ ).

With regards to temperature, adiabatic conditions are assumed and all internal plastic work is converted into temperature change, as per Equation (3.5)[6].

$$
\Delta T=\frac{\overline{\sigma \varepsilon}^{p}}{\rho C_{v}}
$$


Where the effective stress is defined as $\bar{\sigma}=\sqrt{\frac{3}{2} \sigma_{i j} \sigma_{i j}}$, and the effective plastic strain is defined as $d \bar{\varepsilon}^{p}=\sqrt{\frac{2}{3} d \varepsilon_{i j} d \varepsilon_{i j}}$.

\subsubsection{Johnson and Cook Damage Model}

As in many strength models, the $\mathrm{JC}$ failure model calculates a damage ratio given by Equation (3.6). This is done on an element-by-element basis.

$$
D=\sum \frac{\Delta \bar{\varepsilon}^{p}}{\varepsilon_{\text {failure }}}
$$

However, unlike some damage models, the failure accumulation in the JC model does not directly degrade the yield surface [6]. Instead, when the damage parameter, D, reaches 1 fracture occurs, and element stresses are set to, and kept at, zero. The failure strain of the material is defined in Equation (3.7), where $\sigma^{*}$ is the ratio of pressure to effective stress as defined in Equation (3.8) [6].

$$
\begin{aligned}
\varepsilon & =\left[D_{1}+D_{2} \exp \left(D_{3} \sigma^{*}\right)\right]\left[1+D_{4} \ln \left(\dot{\varepsilon}^{*}\right)\right]\left[1+D_{5} T^{*}\right] \\
\sigma^{*} & =\frac{\text { pressure }}{\bar{\sigma}}
\end{aligned}
$$

As in the equation describing the JC flow surface (3.1), JC failure strain (3.7) is broken down into three component effects. These describe fracture strain as a function of hydrostatic tension, material ductility as a function of strain rate, and material ductility as a function of thermal softening [6]. As in the JC flow surface (3.1), parameters $D_{1}, D_{2}, D_{3}, D_{4}, D_{5}$ are experimentally determined. 


\subsubsection{Material Properties}

As the focus of this paper is an investigation of modeling techniques, the work of several other papers was used to establish material properties for analysis. Table 3.1 contains a list of the basic material properties used for simulation, while tables 3.2 and 3.3 contain the material properties used to in Johnson and Cook Material Models.

Tables 3.1, 3.2, and 3.3 contain material property information for five materials. Specifically OHFC Copper, 4340 Steel, Lead, 2024-T351 Al, and Ti (0.06 AL, 0.04 V). The inclusion of 2024-T351 Al, and $\mathrm{Ti}(0.06 \mathrm{AL}, 0.04 \mathrm{~V})$ material properties is the obvious result of their use in the target plates. Leaving the material composition of the bullet to include copper, steel, and lead. The "OHFC" Copper is a generic form of pure copper used in the bullet jacket. The bullet penetrator is composed 4340 Steel, an alloy known for high strength and impact resistance. The second half the bullet interior is composed of a generic lead, which though relatively soft adds the mass of the bullet.

The basic material properties gathered in Table 3.1 are widely available, but were specifically found on Matweb ${ }^{1}$, the online material property database. The material properties found in Tables 3.2 and 3.3 were more difficult to come by. Due to the general difficulty, high cost, and proprietary nature of most material testing finding reliable and consistent material properties was difficult. The availability of Johnson and Cook material and damage properties comes mostly from scholarly

\footnotetext{
${ }^{1}$ http://www.matweb.com
} 
articles on the topic, but rarely are such papers in agreement. So as not to rely on averaged material property data, gathered with inconsistent methodologies, from multiple sources, the most authoritative source was chosen. Specifically an survey of material property data conducted by Los Alamos National Labs [5].

Table 3.1 - Material Properties generated by Matweb

\begin{tabular}{|c|c|c|c|c|}
\hline Material & $\begin{array}{l}\text { Density } \\
{\left[\mathrm{Kg} / \mathrm{m}^{3}\right]}\end{array}$ & $\begin{array}{c}\text { Young's Modulous } \\
{[\mathrm{Pa}]}\end{array}$ & Poisson's Ratio & $\begin{array}{c}\text { Specific Heat } \\
{[\mathrm{J} / \mathrm{Kg} \mathrm{K}]}\end{array}$ \\
\hline OHFC Copper & 8952 & $\begin{array}{ll}1.10 \mathrm{e} 11 \\
\end{array}$ & 0.34 & 383 \\
\hline Lead & 10220 & $1.40 \mathrm{e} 10$ & 0.42 & 100 \\
\hline 4340 Steel & 7830 & $2.05 \mathrm{e} 11$ & 0.29 & 477 \\
\hline 2024-T351 Al & 2770 & $7.31 \mathrm{e} 10$ & 0.33 & 875 \\
\hline $\mathrm{Ti}(0.06 \mathrm{AL}, 0.04 \mathrm{~V})$ & 4427 & $1.14 \mathrm{e} 11$ & 0.34 & 134 \\
\hline
\end{tabular}

Table 3.2 - Johnson and Cook Material Model Properties [5]

\begin{tabular}{c|ccccccc} 
& \multicolumn{1}{|c}{$\begin{array}{c}\text { A } \\
\text { Material }\end{array}$} & $\begin{array}{c}\mathrm{B} \\
{[\mathrm{MPa}]}\end{array}$ & $\mathrm{n}$ & $\mathrm{C}$ & $\mathrm{m}$ & $\begin{array}{c}\text { To } \\
{[\mathrm{MP}]}\end{array}$ & $\begin{array}{c}\mathrm{Tm} \\
{[\mathrm{K}]}\end{array}$ \\
\hline \hline OHFC Copper & $8.96 \mathrm{e} 07$ & $2.91 \mathrm{e} 08$ & 0.31 & 0.025 & 1.09 & 300 & 1355 \\
Lead & $1.03 \mathrm{e} 07$ & $4.13 \mathrm{e} 07$ & 0.21 & 0.003 & 1.03 & 300 & 600 \\
4340 Steel & $7.92 \mathrm{e} 08$ & $5.09 \mathrm{e} 08$ & 0.26 & 0.014 & 1.03 & 300 & 1793 \\
2024-T351 Al & $2.64 \mathrm{e} 08$ & $4.26 \mathrm{e} 08$ & 0.34 & 0.015 & 1.00 & 300 & 775 \\
$\mathrm{Ti}(0.06 \mathrm{AL}, 0.04 \mathrm{~V})$ & $8.61 \mathrm{e} 08$ & $3.30 \mathrm{e} 08$ & 0.34 & 0.012 & 0.80 & 300 & 1905 \\
& & & & & & &
\end{tabular}


Table 3.3 - Johnson and Cook Material Damage Properties [5]

\begin{tabular}{c|ccccc} 
& & & & & \\
Material & D1 & D2 & D3 & D4 & D5 \\
\hline \hline OHFC Copper & 0.54 & 4.89 & 3.03 & 0.014 & 1.12 \\
Lead & 0.25 & 0 & 0 & 0 & 0 \\
4340 Steel & -0.80 & 2.10 & -0.50 & 0.002 & 0.61 \\
2024-T351 Al & 0.13 & 0.13 & 1.50 & 0.011 & 0 \\
Ti (0.06 AL, 0.04 V) & -0.09 & 0.25 & 0.50 & 0.014 & 3.87
\end{tabular}




\section{CHAPTER 4}

\section{RESULTS}

The following section contains both summary statistics and the raw data, generated by the experiment and simulations. Figures 4.17 and 4.18 provide an overview of results of the simulations, compared with the experimental results. Section 4.1.1 contains the raw data points collected during the experiment, while Section 4.1.2 contains photos of the experimental specimens.

\subsection{Experimental Results}

This section contains all experimental velocity data collected (Section 4.1.1), as well as photos of the target plates and cardboard post-impact (Section 4.1.2).

\subsubsection{Chronograph Data}

The complete experimental results for exit velocities in Ti-6Al-4V are presented in Table 4.2. The complete results for exit velocities in 2024 aluminum are presented in Table 4.1. An additional ten rounds were fired, through the chronograph only, to establish an an average entrance velocity as seen in Table 4.4. 
Table 4.1 - M855 Exit Velocities [m/s] (2024 aluminum)

\begin{tabular}{c|cccc} 
& \multicolumn{4}{|c}{ Plate Thickness } \\
Trial & $0.032 "$ & $0.063 ”$ & $0.125 "$ & $0.250 ”$ \\
\hline \hline 1 & 900 & 865 & 803 & 920 \\
2 & 919 & 878 & 810 & 916 \\
3 & 923 & 860 & 777 & 899 \\
4 & 873 & 864 & 764 & - \\
5 & 904 & 878 & 821 & - \\
6 & 947 & 905 & 821 & - \\
7 & 903 & 878 & 811 & - \\
8 & 890 & 914 & 814 & - \\
9 & 913 & 804 & 776 & - \\
10 & 891 & 851 & 788 & - \\
\hline Simulation & 909 & 896 & 872 & 823 \\
Average & 906 & 870 & 799 & 912 \\
STDEV & 21 & 30 & 21 &
\end{tabular}

Table 4.2 - M855 Exit Velocities [m/s] (Ti-4-6)

\begin{tabular}{c|ccc} 
& \multicolumn{3}{|c}{ Plate Thickness } \\
Trial & $0.032 "$ & $0.063 "$ & $0.125 "$ \\
\hline \hline 1 & 911 & 872 & 798 \\
2 & 878 & 860 & 858 \\
3 & 889 & 827 & 864 \\
4 & 901 & 861 & 783 \\
5 & 874 & 832 & 796 \\
6 & 910 & 854 & 784 \\
7 & 921 & 849 & 800 \\
8 & 882 & 836 & 770 \\
9 & 944 & 810 & 740 \\
10 & 863 & 888 & 796 \\
\hline Simulation & 903 & 890 & 858 \\
Average & 897 & 849 & 799 \\
STDEV & 25 & 23 & 37
\end{tabular}


Table 4.3 - M855 Entrance Velocities [m/s] (Ti-4-6)

\begin{tabular}{c|cccccccccc} 
Trial Number & 1 & 2 & 3 & 4 & 5 & 6 & 7 & 8 & 9 & 10 \\
\hline \hline Entrance Velocity & 910 & 908 & 934 & 908 & 949 & 931 & 899 & 897 & 904 & 920
\end{tabular}

Table 4.4 - M855 Entrance Velocities [m/s] (Ti-4-6), statistics

\begin{tabular}{c|cc} 
Trial & Average & STDEV \\
\hline \hline Entrance Velocity & 916 & 17
\end{tabular}




\subsubsection{Photos of Experiment}

A representative set of post impact plate samples are shown in the photos contained within this section. Additionally photos of the post impact cardboard, placed as shown in Figure 2.1, can also be seen in this section.
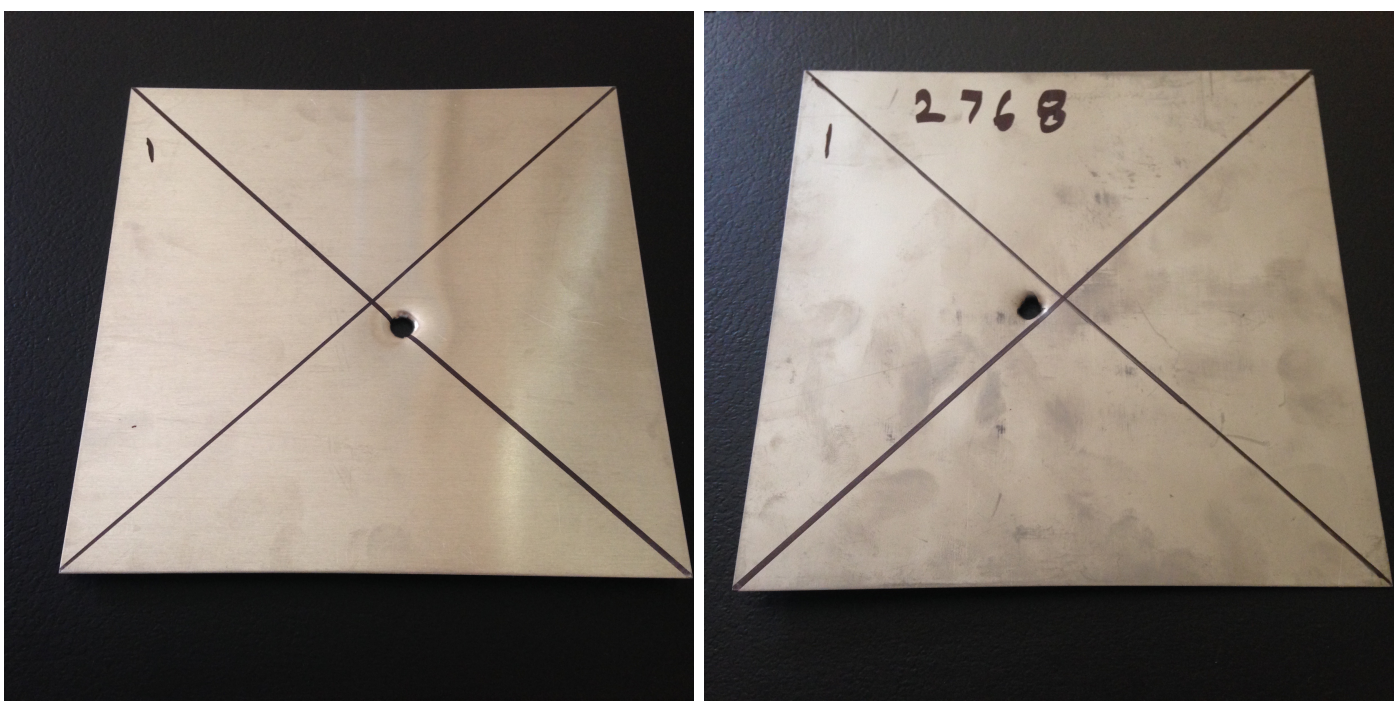

Figure 4.1 - Entrance holes in an aluminum (left) and titanium (right) 0.032 " thick plates. 

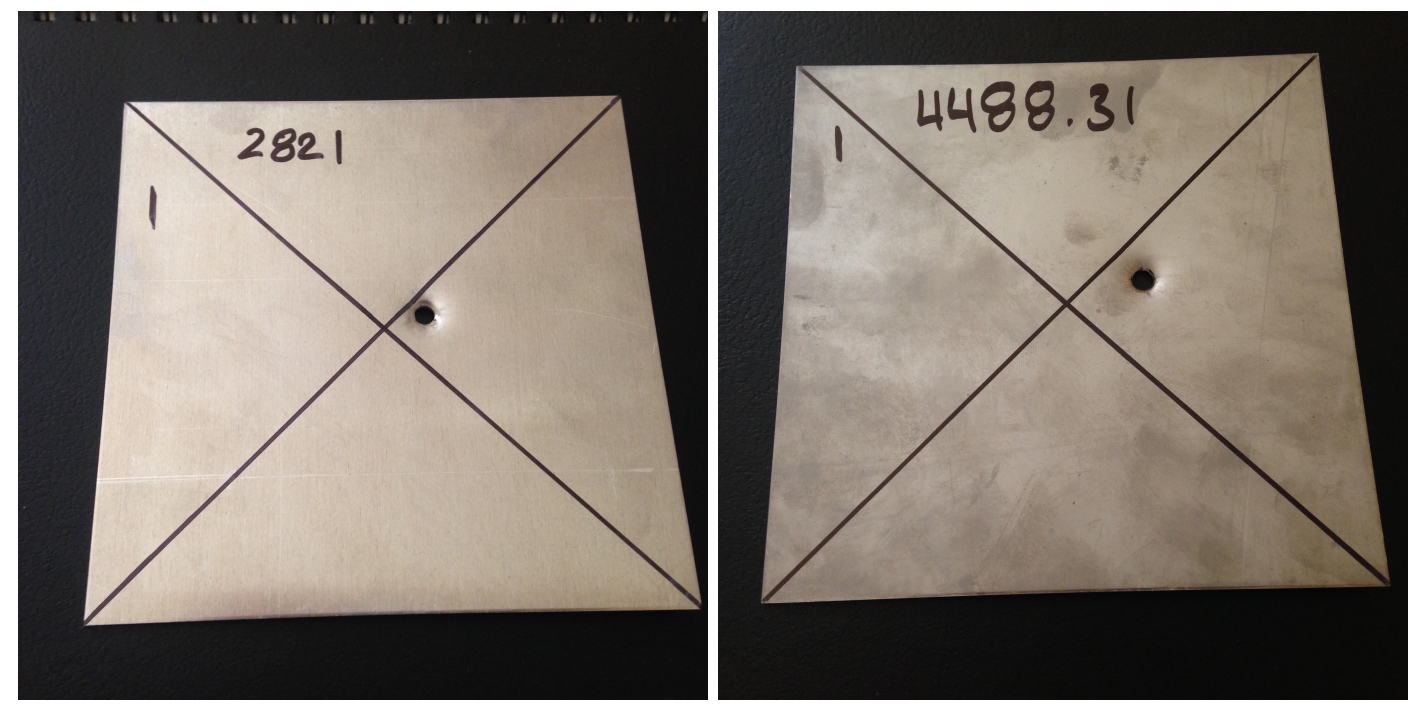

Figure 4.2 - Entrance holes in an aluminum (left) and titanium (right) 0.063 " thick plates.
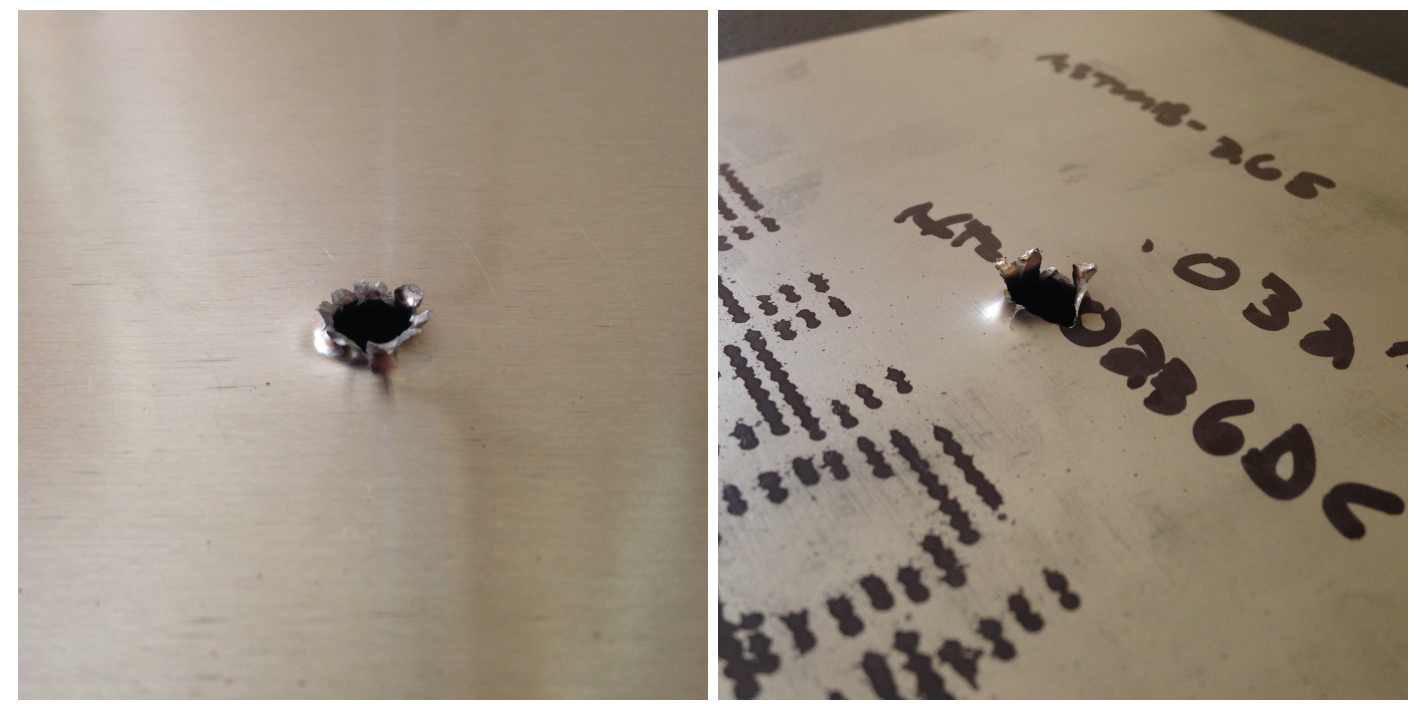

Figure 4.3 - Exit holes in an aluminum (left) and titanium (right) 0.032 " thick plates. 

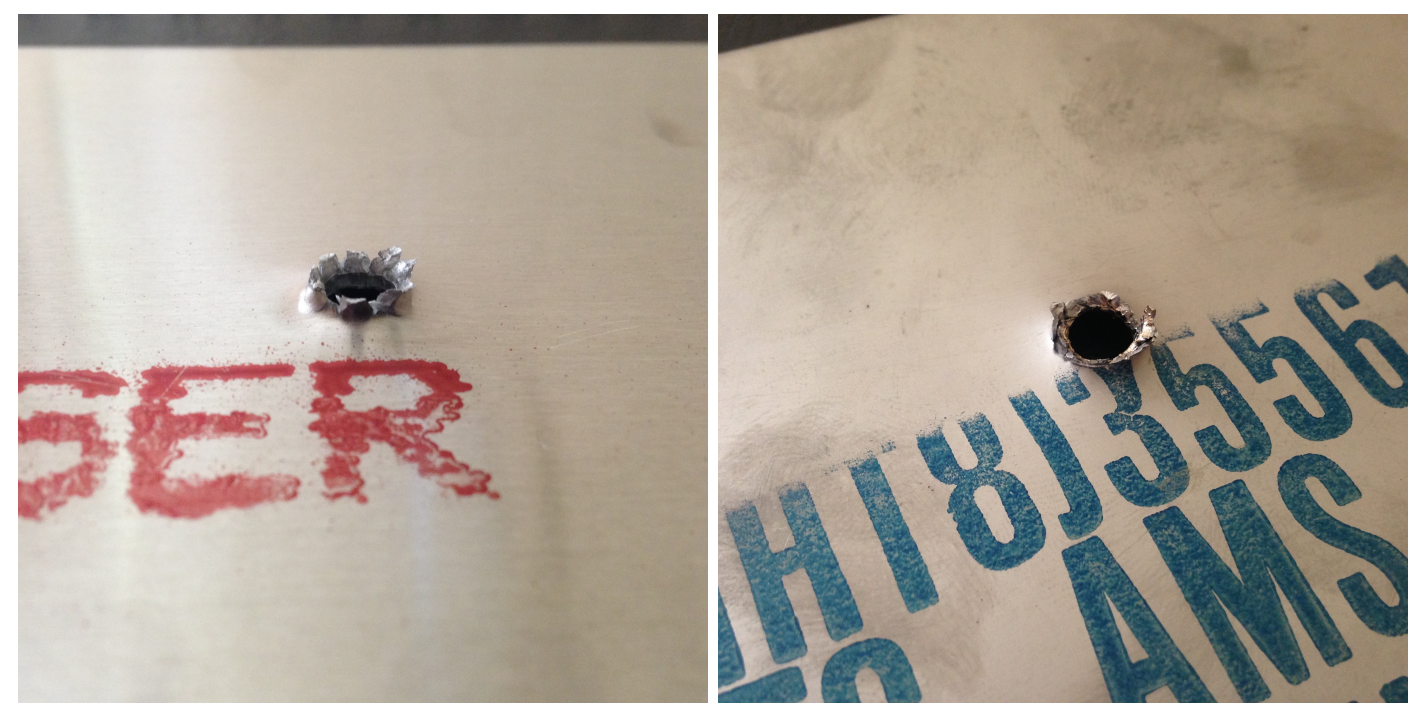

Figure 4.4 - Exit holes in an aluminum (left) and titanium (right) 0.063 " thick plates.
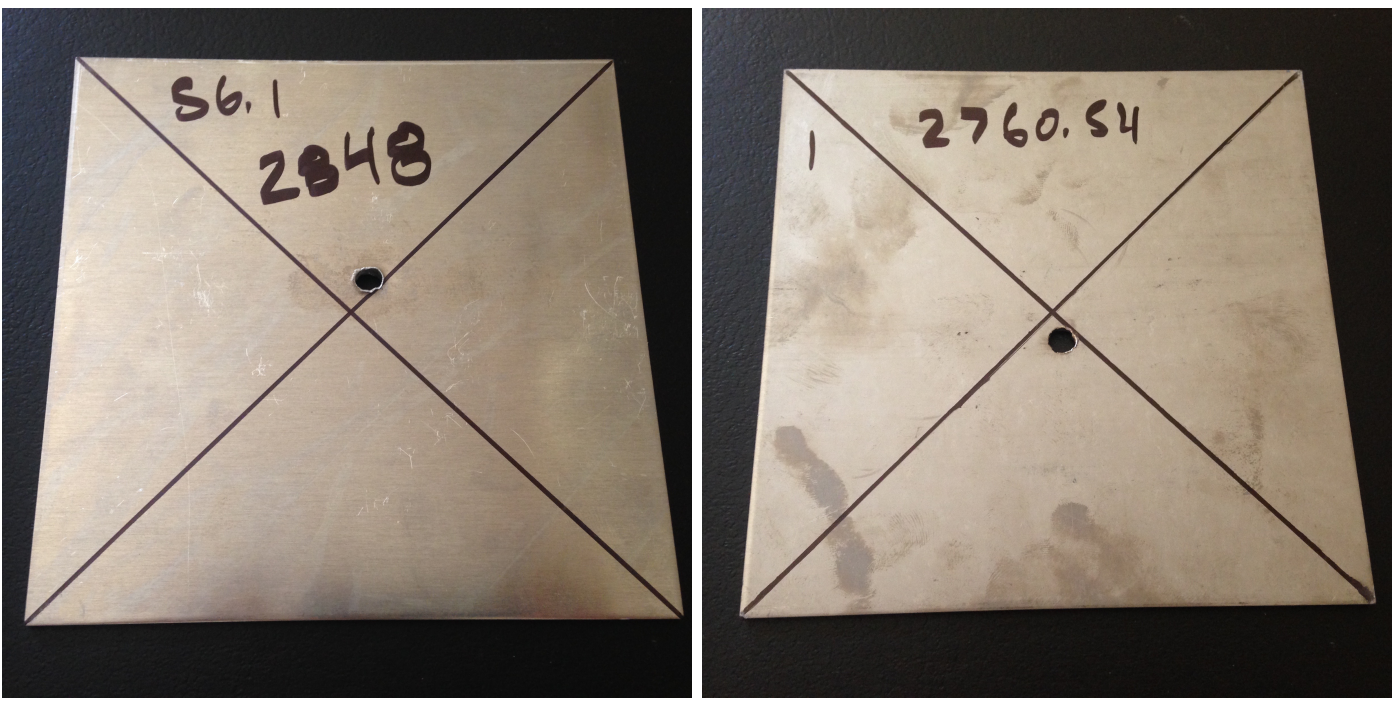

Figure 4.5 - Entrance holes in an aluminum (left) and titanium (right) 0.125 " thick plates. 

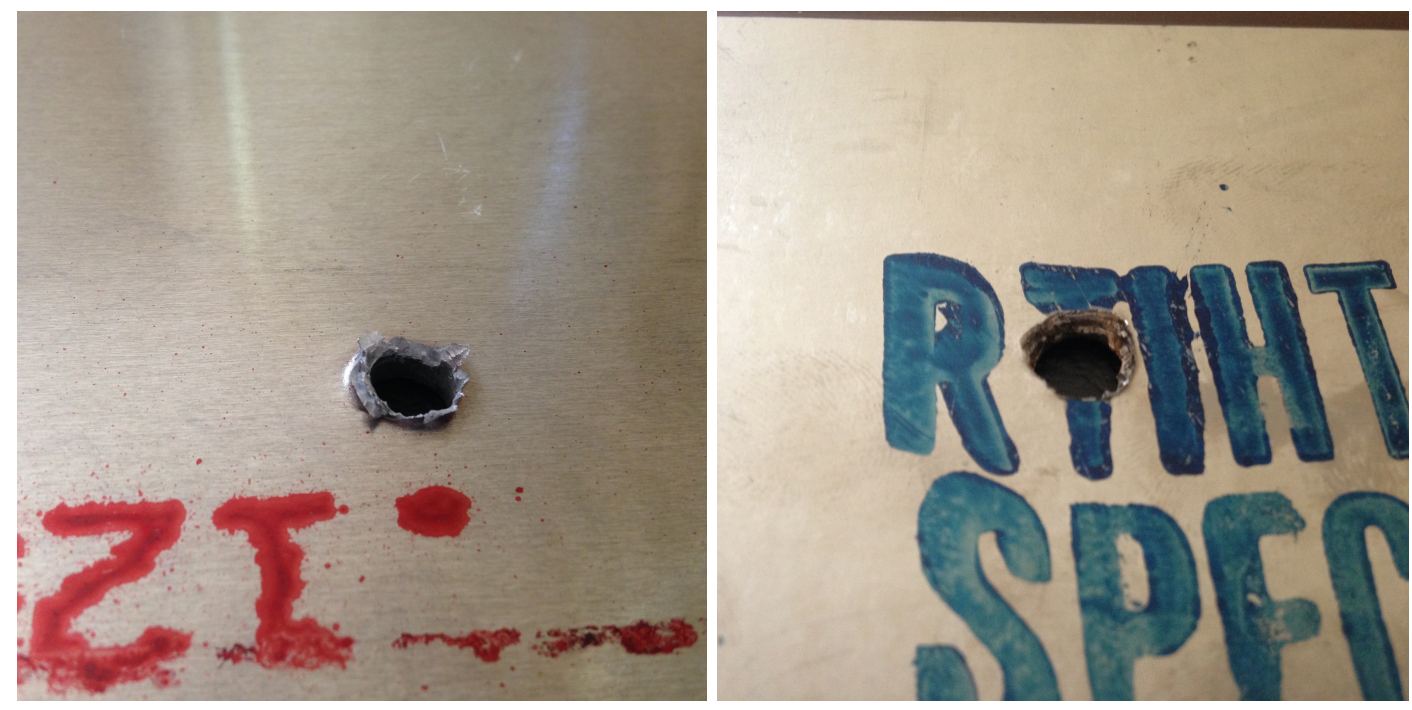

Figure 4.6 - Exit holes in an aluminum (left) and titanium (right) 0.125 " thick plates.

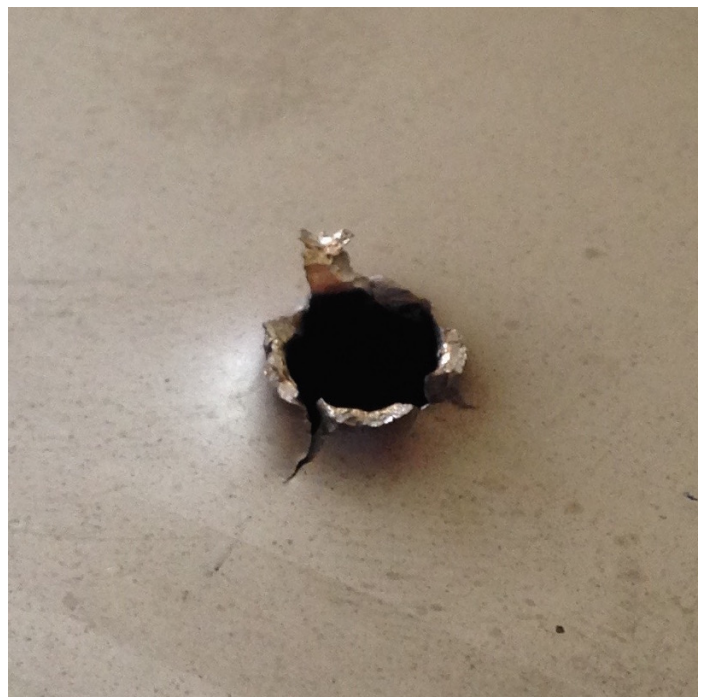

Figure $4.7-0.032$ " titanium plate displaying brittle material petaling behavior. 


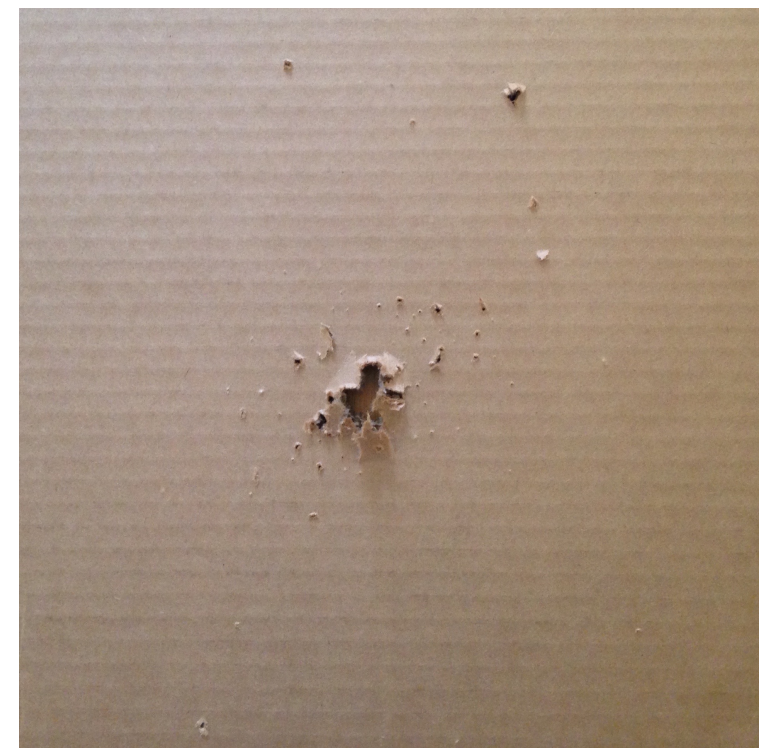

Figure 4.8 - M855 shrapnel spread pattern created after passing through 0.063" aluminum plate.

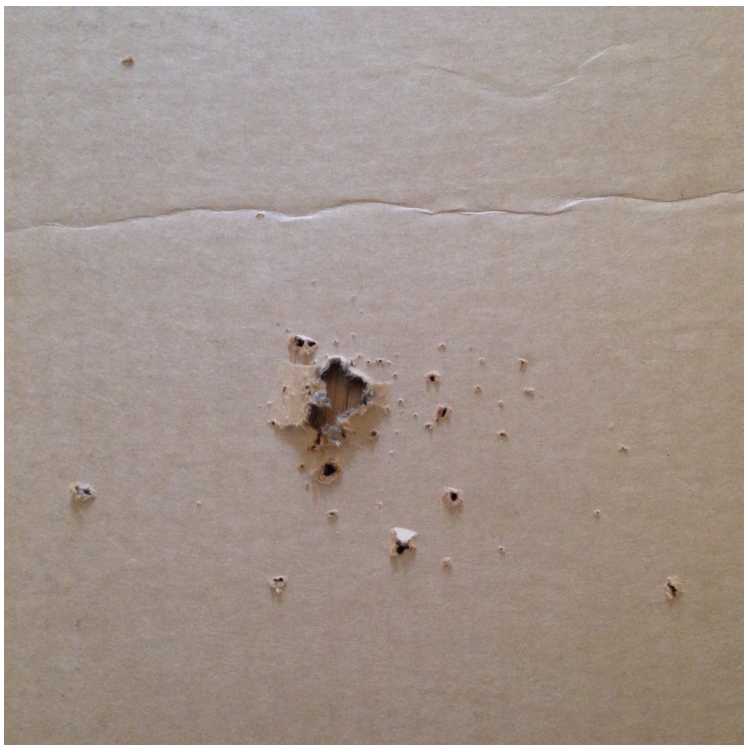

Figure 4.9 - M855 shrapnel spread pattern created after passing through 0.063 ” titanium plate. 


\subsubsection{Discussion/Observations of Experimental Results}

Aside from the raw exit velocity data presented in Table 4.1 and 4.2, several observations about the photos presented in Section 4.1.2 can be made. Firstly, the entrance holes (Figures 4.1 and 4.2 ) in the titanium and aluminum plates of thicknesses 0.032 " and 0.063 " are very similar, while their exit holes look significantly different (Figures 4.3 and 4.4). The aluminum plate of 0.032 " and 0.063 " thickness displays distinctive petaling formations, characteristic of thin plate impacts [11]. The titanium specimens do not appear nearly as symmetrical, and do not present as many individual petals; asymmetry being characteristic of failure in brittle materials [11]. An additional example of the titanium fracture can be seen in Figure 4.7.

Though the petaling formations described do appear different, both materials display the characteristic formations to some degree, implying petaling is present in both cases. However, from both the velocity data and photos, it appears that 0.063 " appears to be a threshold (or transition point) beyond which as shift in the petaling behavior is observed. Where two distinctly different results are observed in samples of thickness 0.063 " on each side of this apparent threshold. This transition is indicative of a shift from thin plate petaling behavior, to thick plate fragmentation. This shift in behavior is further seen in Figures 4.8 and 4.9. These shrapnel pattern photos, show that in plate thicknesses 0.125 " and above, diameter of the shrapnel spray area grows. This indicates that, at this plate thickness, the bullet and or plate have undergone large deformations.

In the velocity data alone, this behavior is no immediately apparent. The 0.25 " aluminum plate exit velocities, shown in Table 4.1, are incomplete, as the chronograph was unable to produce valid readings due to fragmentation debris. Instead 
registering exit velocities higher than the initial entrance velocity. It is most likely that the highly fragmented plate and bullet were no longer capable of properly triggering the chronograph's light sensors, yielding inaccurate results. This may have been due to spalling effects, ejecting small fragments of plate at high velocities ahead of the bullet. Access to a high-speed camera for future experiments is highly desirable.

\subsection{Modeling Results}

\subsubsection{Velocity Results}

In order to facilitate quick comparison between model and experiment, Tables 4.7 and 4.6 summarize exit velocity in rigid body and nonrigid body simulations. The velocities reported are the "lumped mass" velocities for the steel core, in the direction of travel orthogonal to the plate. Figure 4.10 and 4.11 show typical velocity plots over the duration of the simulation. Simulations were run until steel core and lead core velocities plateaued, their difference in velocities less than $1 \mathrm{~m} / \mathrm{s}$.

Comparing Tables 4.7 and 4.6, it is interesting to note the minimal difference between rigid and nonrigid core impacts with the target plate. The difference between the two predicted velocities as plate thickness increases. This is expected, since bullet deformation is minimal in the thin plate experiments (Figure 4.19). This suggests suggests future attempts to better replicate petaling behavior would be successful with two changes: with drastically increased plate mesh density and with the bullet modeled as a rigid body (this done to reduce computational expense).

Upon impact, the components of both models compress and then expand. However, in the rigid core model the stiffness of the lead and steel core components is 
increased, and thus the frequency of oscillation is also increase. This is an unrealistic modeling result, but since the final velocity is relatively unchanged the effects appear negligable.

Table 4.5 - Total Deformable Element Count for Various Levels of Mesh Refinement

\begin{tabular}{c|ccc|ccc} 
& \multicolumn{3}{|c}{ Rigid Mesh } & \multicolumn{3}{c}{ Non-Rigid Mesh } \\
Plate Thickness [in] & Low & Medium & High & Low & Medium & High \\
\hline \hline 0.032 & 273 & 1245 & 7137 & 3245 & 4217 & 10109 \\
0.063 & 273 & 1360 & 1968 & 3245 & 4332 & 4940 \\
0.125 & 273 & 3790 & 5685 & 3245 & 6762 & 8657 \\
0.250 & 273 & 1728 & 3936 & 3245 & 4700 & 6908
\end{tabular}

Table 4.6 - Simulated Fully Deformable Exit Velocities in $\mathrm{m} / \mathrm{s}$, With Varying Element Count

\begin{tabular}{c|ccc|ccc} 
& \multicolumn{3}{|c}{ Aluminum } & \multicolumn{3}{c}{ Titanium } \\
Plate Thickness [in] & Low & Medium & High & Low & Medium & High \\
\hline \hline 0.032 & 907 & 908 & 909 & 903 & 904 & 903 \\
0.063 & 890 & 897 & 896 & 879 & 891 & 890 \\
0.125 & 869 & 870 & 872 & 859 & 847 & 858 \\
0.250 & 822 & 836 & 823 & 909 & 909 & 909
\end{tabular}




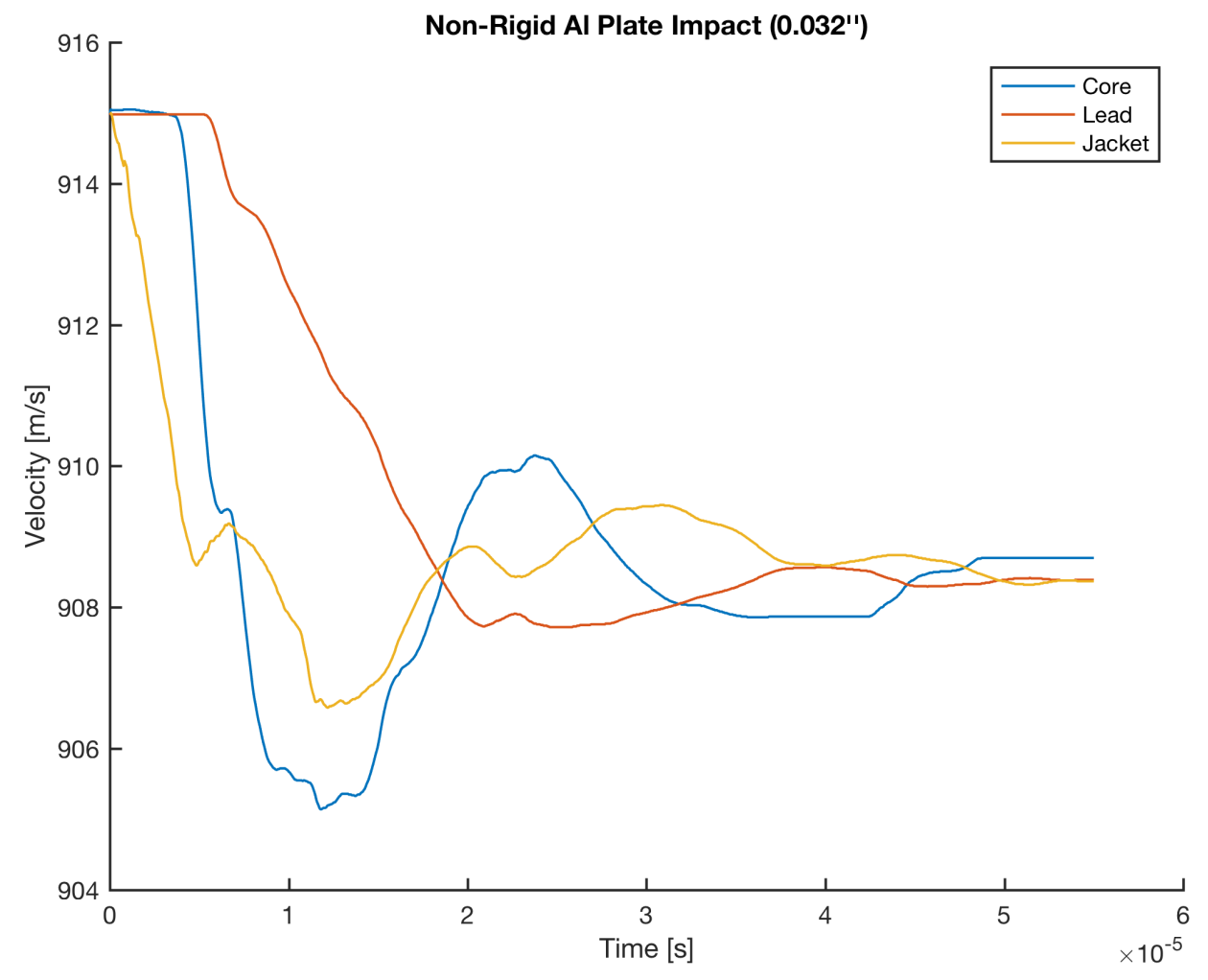

Figure 4.10 - Part velocities for simulation of impact with 0.032 " aluminum plate, in which bullet core and lead were modeled as rigid.

Table 4.7 - Simulated Rigid Core Exit Velocities in m/s, With Varying Element Count

\begin{tabular}{c|ccc|ccc} 
& \multicolumn{3}{|c}{ Aluminum } & \multicolumn{3}{c}{ Titanium } \\
Plate Thickness [in] & Low & Medium & High & Low & Medium & High \\
\hline \hline 0.032 & 907 & 909 & 909 & 904 & 903 & 905 \\
0.063 & 895 & 899 & 895 & 885 & 893 & 892 \\
0.125 & 875 & 875 & 883 & 861 & 866 & 876 \\
0.250 & 840 & 836 & 841 & 909 & 909 & 909
\end{tabular}




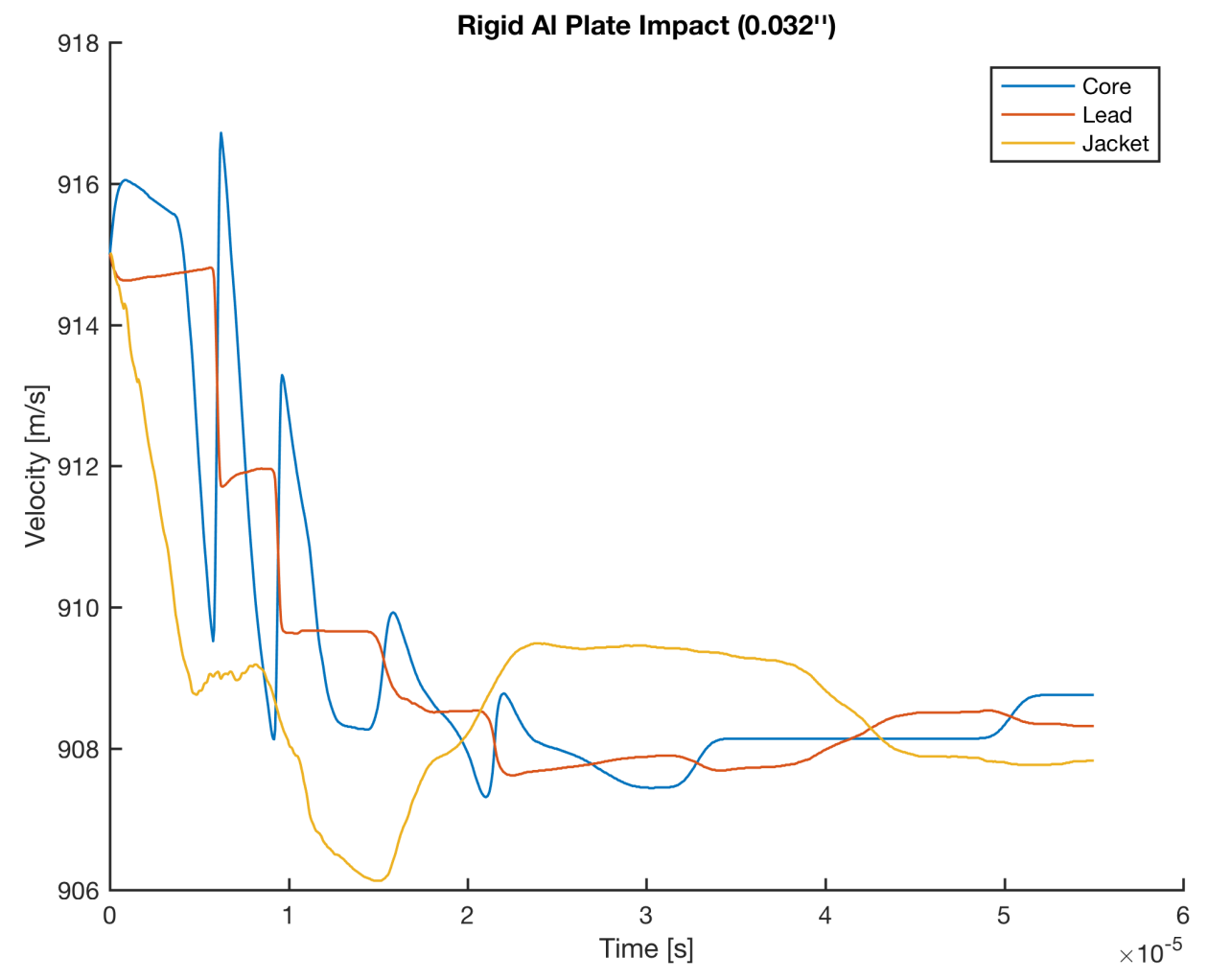

Figure 4.11 - Part velocities for simulation of impact with 0.032 " aluminum plate, in which all components were modeled as fully deformable. 


\subsubsection{Conservation of Energy and Time step Selection}

An analysis of energy balance is always important when verifying an explicit model. However, it is even more so when element erosion is a significant modeling parameter. As discussed in Section 3.2, Model Formulation, time step based erosion was used to stop trapped elements from creating unworkably long runtimes.

The element erosion time step was set to $2 e-9$ seconds, well below the contact time step size. It thus dictated overall simulation time step, an effect readily apparent in Figure 4.13, which depicts the simulation time step for the 0.250 " aluminum plate. The same simulation's energy balance is shown in Figure 4.24, while the effect of erosion on the system energy balance for all simulations is summarized as a percent change in Tables 4.8 and 4.9.

Erosion time steps, which allowed the simulation to be dictated by contact time step, gave unrealistically high velocities. The cause of this was visually obvious: as the elements in the plate simply disappeared upon impact, undergoing little deformation, and allowing the bullet to pass through the plate unimpeded. The effect on the system's energy balance was also clear, as total system energy was not conserved. Conversely, decreasing the erosion time step by an order of magnitude to $2 \mathrm{e}-10$ created equally unrealistic results; allowing elements to become so deformed that they no longer functioned. In addition decreasing the time step size to $2 \mathrm{e}-10$ would have resulted in drastically increased runtimes. Element erosion time, time step size, was thus chosen through trial and error to be a value balancing the limits of element deformation and lengthened runtimes against outsize 
effects to the systems energy balance.

The relationship between the contact time step size and transfer of energy between kinetic and internal is notable. As the elements in the plate and bullet begin to deform, kinetic energy is converted to internal energy, and elemental stiffnesses increase. This effectively reduces contact time step size, which is proportional to $\sqrt{\frac{k}{m}}$ [certa sim reference manual]. This relationship is reflected in Figures 4.24 and 4.13 , between $0.6 e-5$ and $2 e-5$ seconds, during which time the bullet comes into contact with, and then passes through, the plate.

There are some risks present when using energy balance to validate time step size, principally seen in models containing element erosion. In these models a relatively small number of elements, which represent an equally small amount of total system energy, have an outsized effect on simulation results. One would expect to see this in simulations focusing on specific types of penetrator tips and exotic materials. This phenomenon highlights one of the greatest difficulties in ballistic model meshes: capturing the conical geometry of the bullet tips, requiring reduced element sizes. This reduces time step size, and in turn, reduces the mesh density of the bullet, as one moves away from the tip. The problem of this increased mesh density at the tip is exacerbated, particularly if there is a great disparity in stiffness between items coming into contact, as contact penetrations can occur.

In simulations of a standard M855 bullet, it is assumed the bullet penetration is driven primarily by the initial kinetic energy, not by special geometries and materials. Thus, the high degree of element deletion at initial contact is deemed acceptable. This is evident in Figure 4.13. This deletion occurs in the smallest elements at the bullet tip, and has a negligible affect on energy balance. 


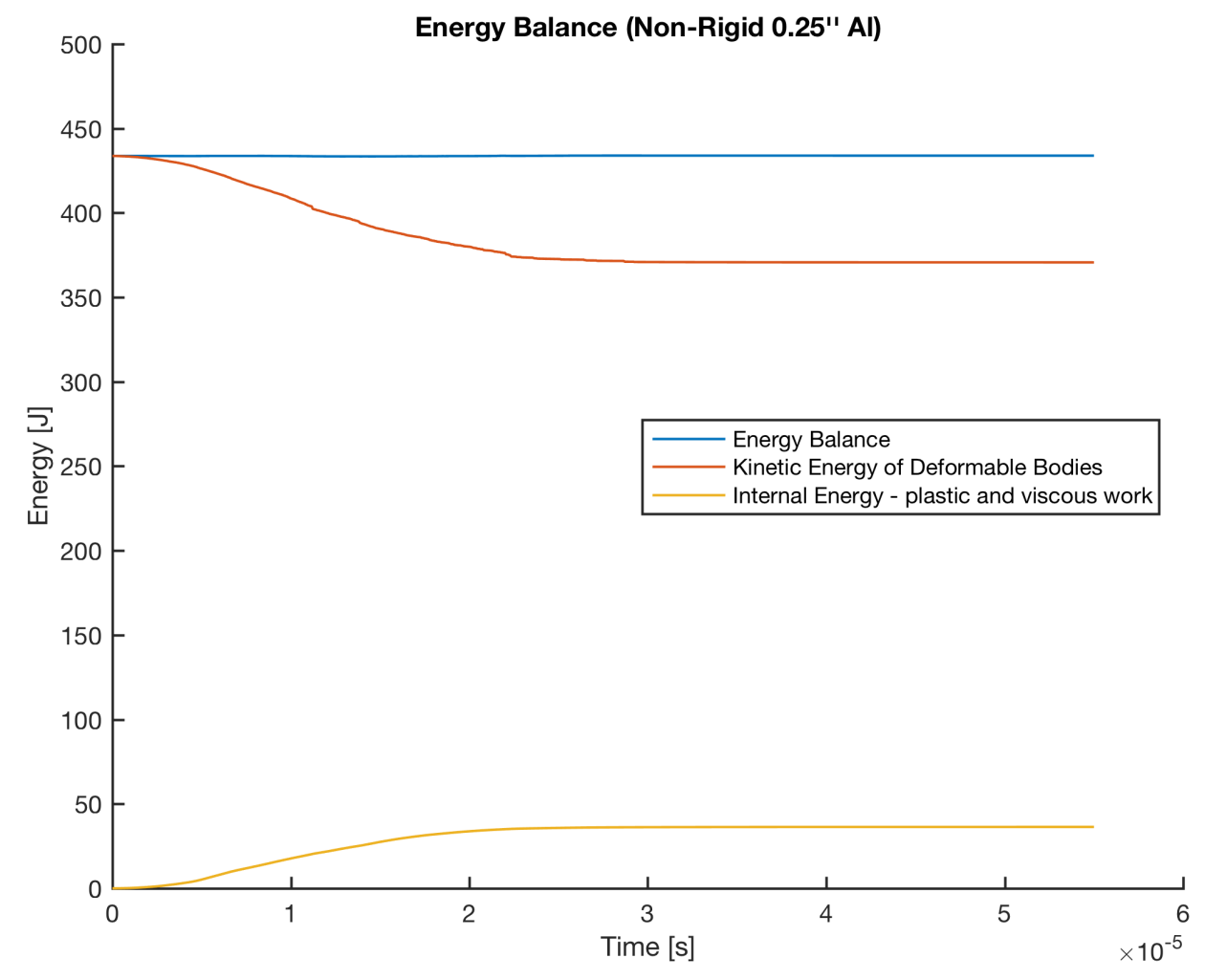

Figure 4.12 - Energy balance results for simulation of fully deformable impact with 0.25 " aluminum plate. 


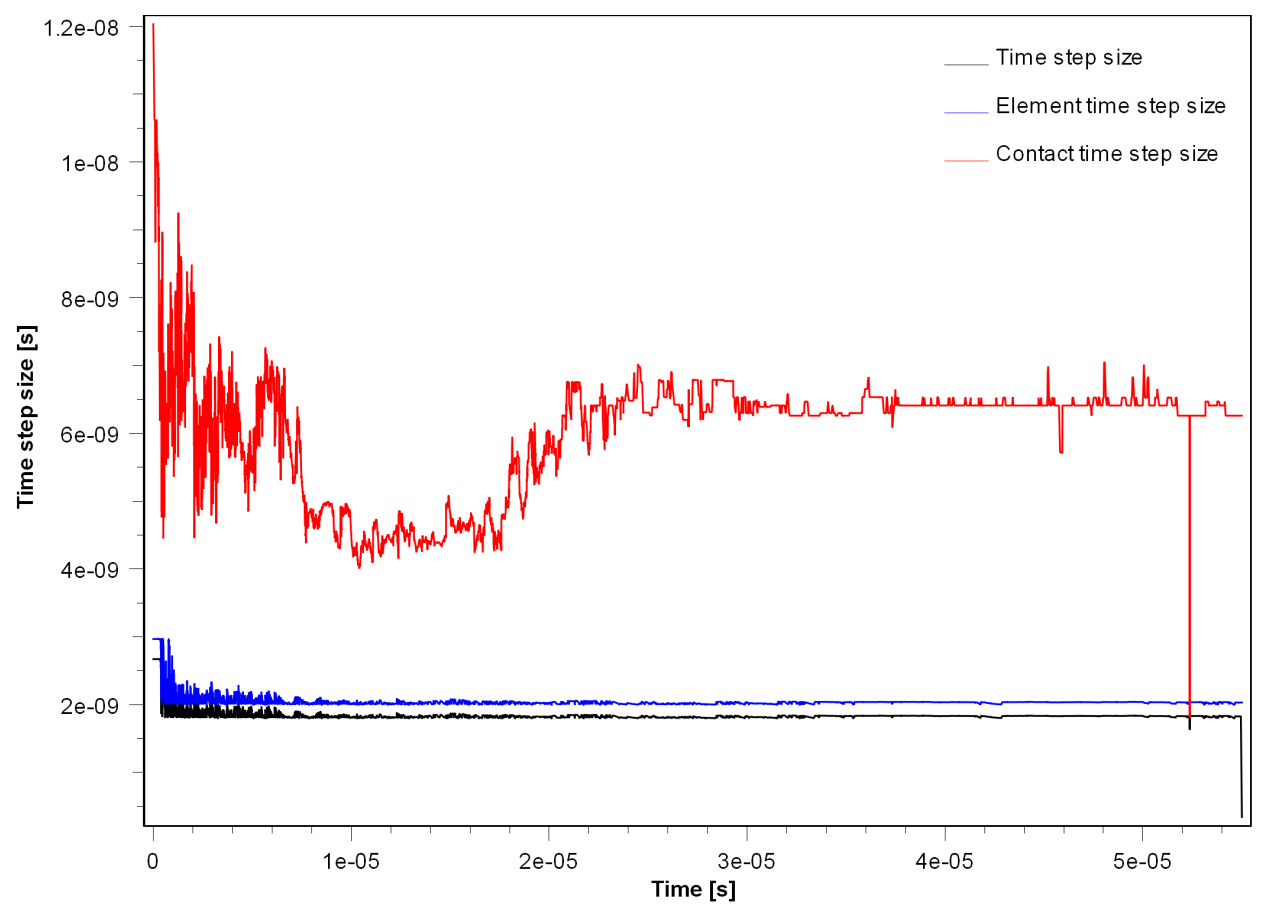

Figure 4.13 - Time step results for simulation of fully deformable impact with 0.25 " aluminum plate.

Table 4.8 - Percent change in energy balance for rigid body simulations, With Varying Element Count

\begin{tabular}{c|ccc|ccc} 
& \multicolumn{3}{|c}{ Aluminum } & \multicolumn{3}{c}{ Titanium } \\
Plate Thickness [in] & Low & Medium & High & Low & Medium & High \\
\hline \hline 0.032 & 0.04 & 0.04 & 0.04 & 0.06 & 0.06 & 0.04 \\
0.063 & 0.05 & 0.03 & 0.03 & 0.09 & 0.03 & 0.03 \\
0.125 & 0.06 & 0.12 & 0.29 & 0.09 & 0.12 & 0.26 \\
0.250 & 0.29 & 0.12 & 0.42 & 0.04 & 0.04 & 0.04
\end{tabular}

Table 4.9 - Percent change in energy balance for non rigid body simulations, With Varying Element Count

\begin{tabular}{c|ccc|ccc} 
& \multicolumn{3}{|c}{ Aluminum } & \multicolumn{3}{c}{ Titanium } \\
Plate Thickness [in] & Low & Medium & High & Low & Medium & High \\
\hline \hline 0.032 & 0.04 & 0.04 & 0.04 & 0.06 & 0.05 & 0.06 \\
0.063 & 0.09 & 0.02 & 0.05 & 0.06 & 0.04 & 0.04 \\
0.125 & 0.04 & 0.13 & 0.30 & 0.45 & 0.06 & 0.33 \\
0.250 & 0.57 & 0.12 & 0.76 & 0.04 & 0.04 & 0.04
\end{tabular}




\subsubsection{Mesh Convergence}

Mesh quality and convergence in multi-part, explicit simulations difficult to establish. The number of mesh permutations grows exponentially with the number of parts considered. Varying total element count while maintaining mesh quality increases the challenge. In complex geometries, such as those in a multi-material bullet, high element counts are required simply to capture the underlying geometry of the model. Increasing mesh density in these areas provides little added value, especially in regions undergoing minimal deformation (the back of the bullet). Increasing mesh density in the target plate - an area experiencing outsize deformations - has a much greater affect on simulations results.

The problem of mesh convergence was consequently tackled in two steps. Effects of mesh density were first studied in the target plate only. In this scenario, the target plate and bullet jacket were fully modeled, while the bullet core was made wholly rigid. Plate mesh density was then assessed at three levels of refinement: low, medium, and high. In the second set of simulations, plate mesh density was again varied, with the bullet and plate modeled as fully deformable.

To simplify the number of mesh permutations required to establish convergence, the study was divided into rigid and non-rigid simulations. This allowed the plate's mesh to be studied separately from the bullet's. It also allows the mesh density of the bullet to be drastically reduced - effectively eliminating, in fact - without losing the underlying objects geometry.

Since the fully deformable versions undergo little deformation anyway, in simulations with plate thicknesses of 0.032 " and 0.063 ", it is not surprising to see that 
rigidly modeled penetrators had little effect on bullet exit velocity. In fact, the main effect on exit velocity was previously covered in Section 4.2.1, which covers the increased oscillations of the rigid core upon impact. Even in plates of greater thickness, where the bullet core experiences greater deformations, the effect of mesh refinement on the plate and bullet core is minimal.

The results of the mesh convergence studies, across all models, are presented in Figures 4.14, 4.15, and 4.16. Variation in exit velocities is presented as a normalized percent change in order to facilitate comparison of mesh refinement effects across plate thicknesses. Refinement had the smallest effects on thin plate models, resulting in exit velocities that change by less than a percent. In thicker plates element count had a greater effect, though variation was still less than two percent. 


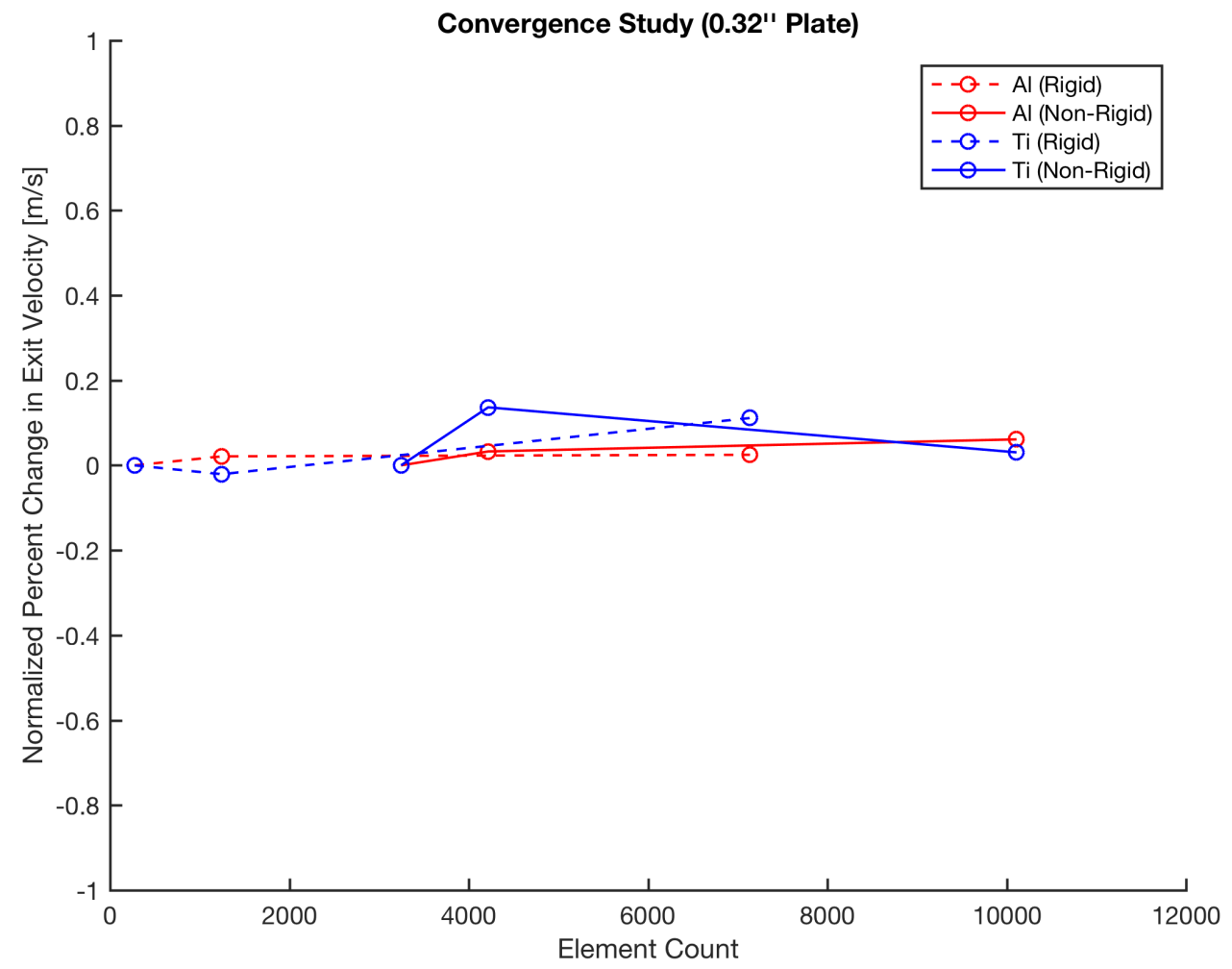

Figure 4.14 - Convergence study of the effects of mesh density on 0.32 " plates plate impacts. 


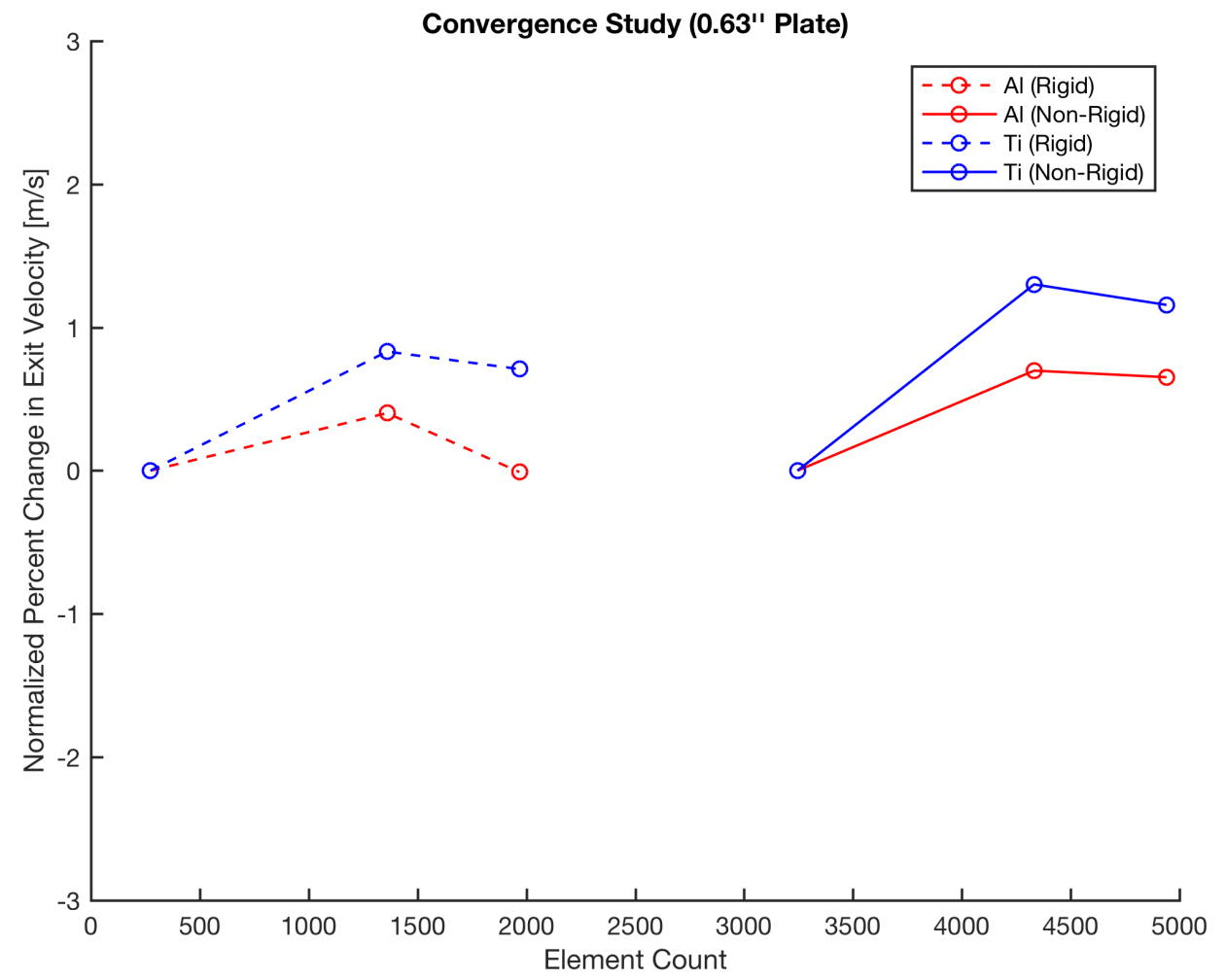

Figure 4.15 - Convergence study of the effects of mesh density on 0.63 " plates plate impacts. 


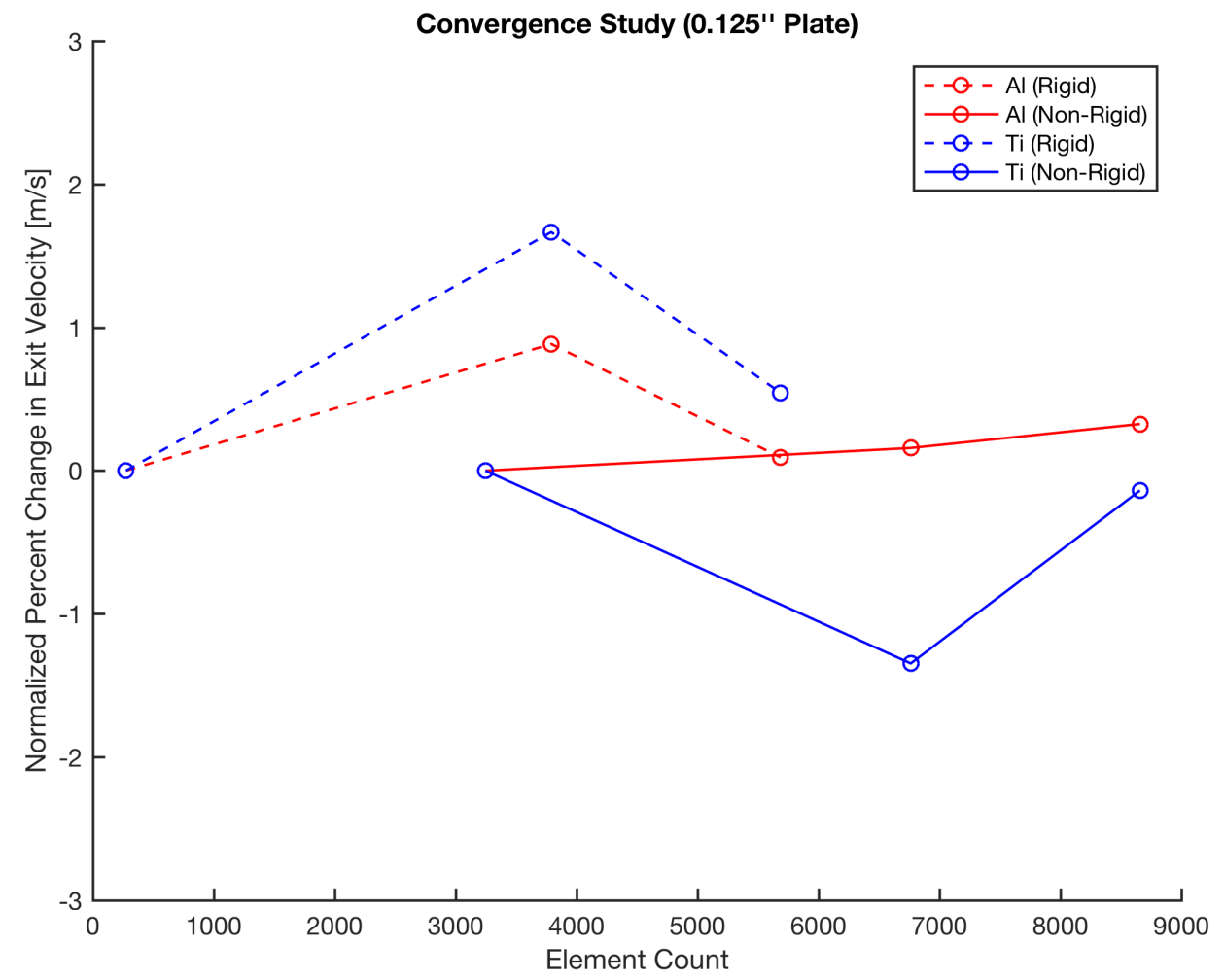

Figure 4.16 - Convergence study of the effects of mesh density on 0.125 " plates plate impacts. 


\subsection{Experimental and Simulation Results}

In comparing observed and simulated results, a short summary of available information is first made. Measurements of bullet velocities are reported in Section 4.1.1, while photos of post-impact target plates with shrapnel spray patterns are shown in Section 4.1.2. A comparison of simulated and experimental exit velocity data is seen in Figures 4.17 and 4.18 .

In both Figures (4.17 and 4.18) it is obvious that simulated and experimental results did not always perfectly align. The trend of both simulated and experimental results begins in general agreement, but with increasing plate thickness simulated results diverge from experimental results.

From the the perspective of a system's energy balance, it is apparent that in simulated impacts, more of the bullet's initial kinetic energy remains with the bullet. Based on this assessment, factors contributing to the simulation's divergence from reality can be classified in two categories. Errors introduced by improper model parameters, and errors introduced by fundamental failings in the physics of the FEA model.

The simplest explanation for the mismatch between results is improper modeling parameters. As discussed in the Section on material properties (Section 3.4.3), research on specific material properties revealed a great amount of inconsistency even among reputable sources. Furthermore, the material properties listed in Section 3.4.3 are not perfectly indicative of the sample bullet, which consists of unlisted (possibly proprietary) alloys. 
However, to say that the whole of the disagreement between simulation and experiment is due to inaccurate material property information is disingenuous. Even in simulations which exactly replicate material property experiments, there is some discrepancy in the results. These disagreements are due to assumptions inherent to the model itself.

One such assumption discussed in Section 3.2 is that the erosion of elements is inherently a non-physical property of the model. Though node splitting was used, and based on the Johnson and Cook damage model, time step based erosion was still necessary (Section 4.2.2). Although the effects on system energy balance were nonexistent, time step erosion likely still had an effect on simulation outcome (see Table 4.8 and 4.9). Presently, mitigation of these effects is not possible without the use of more powerful computers. A more refined mesh and a smaller time step tolerance would be required to combat both numerical instabilities that result in infinitely hardened elements, and to prevent the non-physical effects of element erosion.

The fact that simulated and experimented results diverge with plate thickness is not surprising, since greater thicknesses result in greater deformation (i.e. more interaction). The difficulty associated with accurate representation of larger deformations was acknowledged in Section 3.1, as modeling this scale of deformation is an area of open research.

Mesh density is also a likely contributor to the divergence of results. As discussed in Section 3.3, mesh density would ideally be held constant across all plate thicknesses. However, at the same mesh density, larger plates require more elements to represent increased part volume. Thus, available computational power limits the 
ability of maintain equivalent mesh densities. This issue was addressed in detail in Section 4.2.3, via mesh convergence studies. Though, as with time step based erosion, further mesh refinement would likely have had a beneficial effect.

Somewhat more qualitatively, comparisons between Figures 4.19, 4.20, 4.21, and 4.22 show increased fragmentation in the bullet core with increasing plate thickness. This, combined with the fact that larger plates simply have more material to fragment, indicates that the impact simulations produce a trend similar to that observed in the shrapnel pattern photos (Figures 4.8 and 4.9).

An attempt to replicate the petaling formations discussed in Section 4.1.2 was made with highly local mesh refinement near the point of impact. These attempts were generally unsuccessful; likely due to a lack of computational power. However, further examination of Figures 4.19 through 4.22 does reveal some semblance of this phenomena though to a lesser extent. In Figures 4.19, 4.20, 4.21 a large portion of the plate can be seen peeling away in a petal-like formation. However, in the plate of thickness 0.250 ", the petaling phenomena is much less apparent. This phenomena was observed in Section 4.1.2, though the disappearance of thin plate petaling occurred plates only $0.125 "$ thick. 


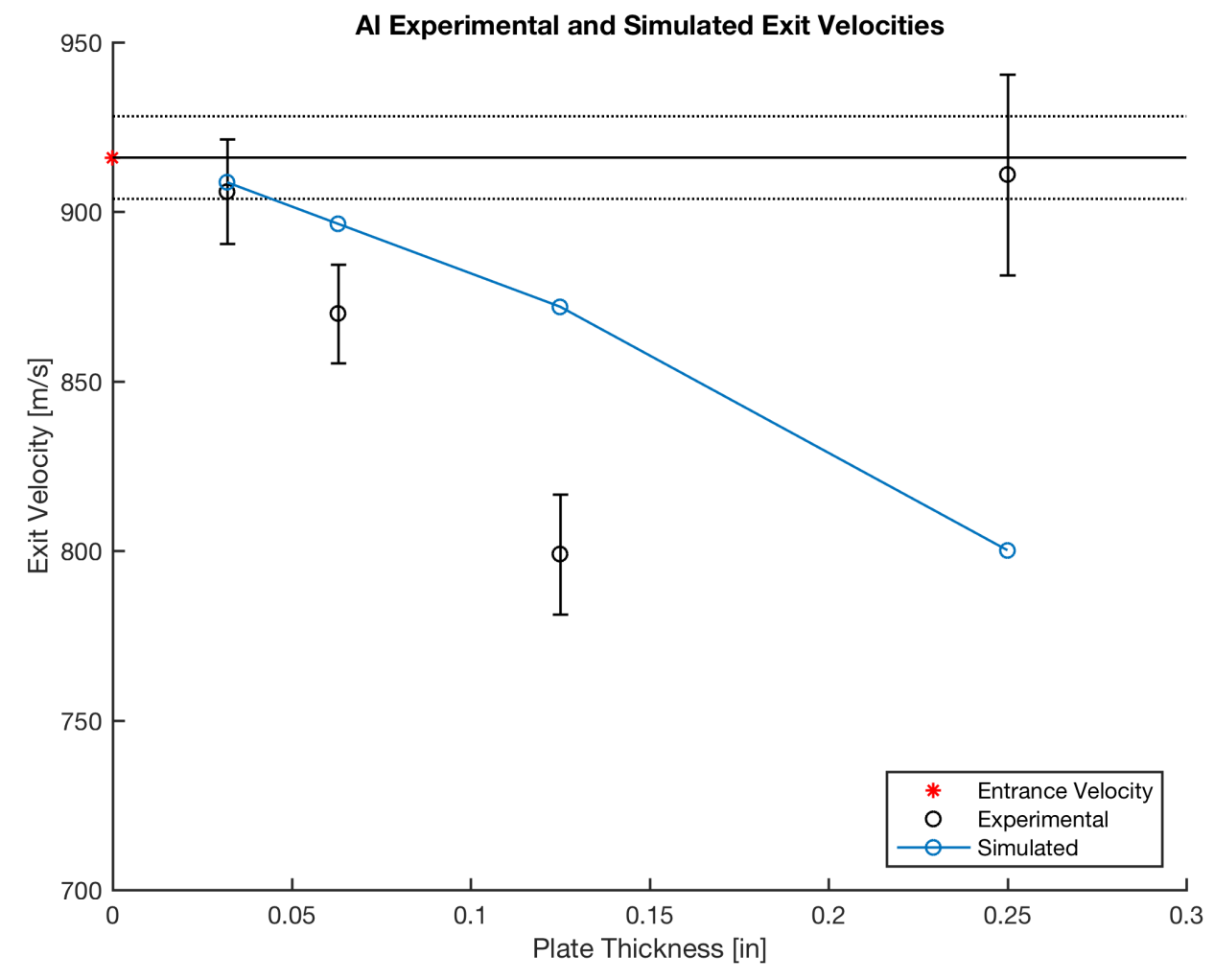

Figure 4.17 - Comparison of experimental and simulated Results of aluminum plates 


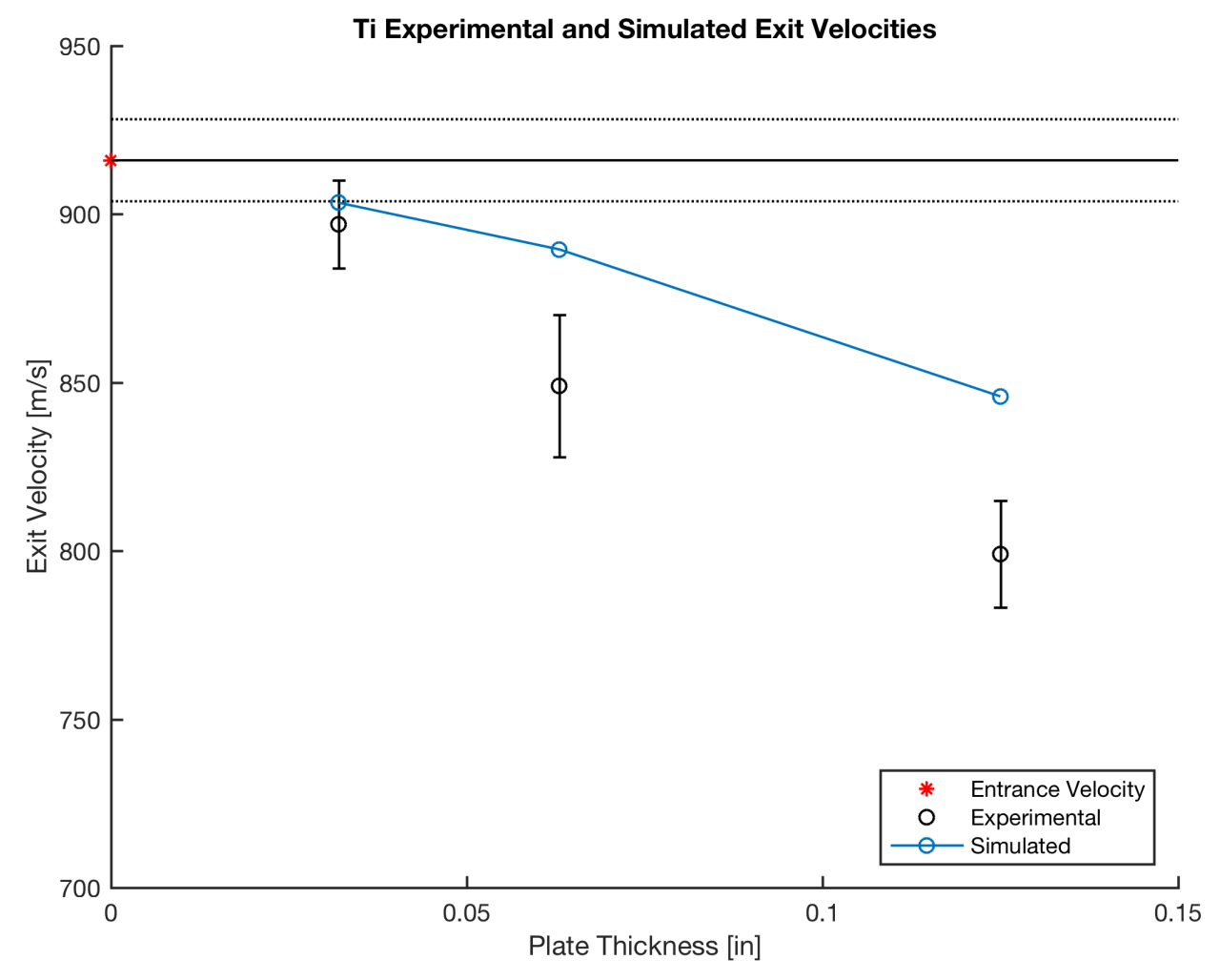

Figure 4.18 - Comparison of experimental and simulated Results of titanium plates

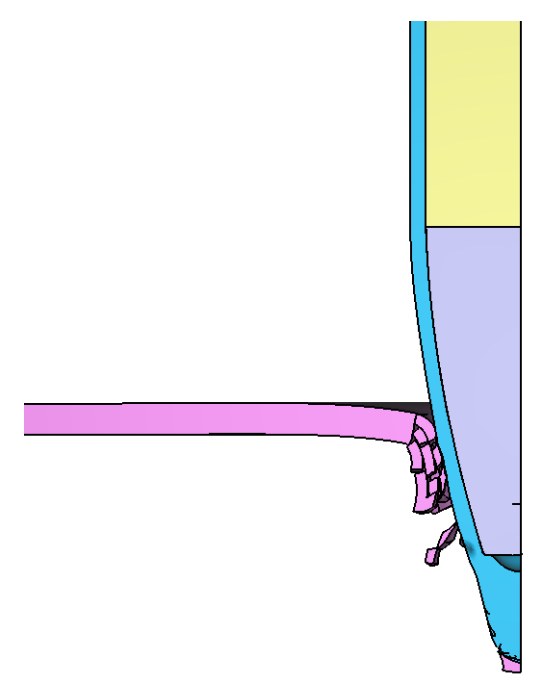

Figure 4.19 - Simulated impact of an M855 bullet with 0.032" thick aluminum plate, represented with a high density mesh. 


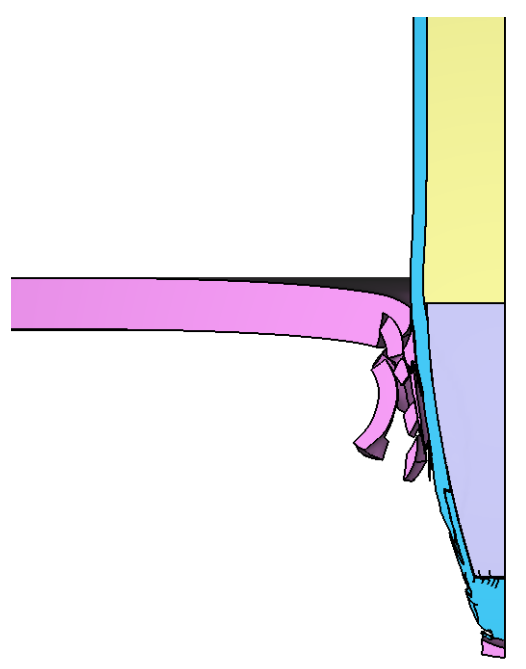

Figure 4.20 - Simulated impact of an M855 bullet with 0.063 " thick aluminum plate, represented with a high density mesh.

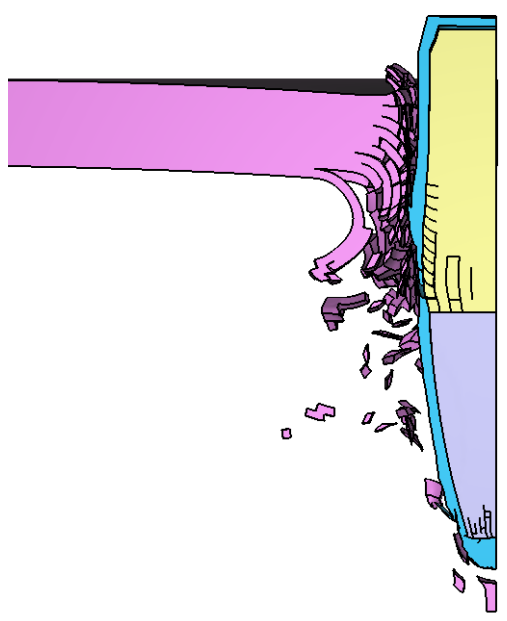

Figure 4.21 - Simulated impact of an M855 bullet with 0.125 " thick aluminum plate, represented with a high density mesh. 


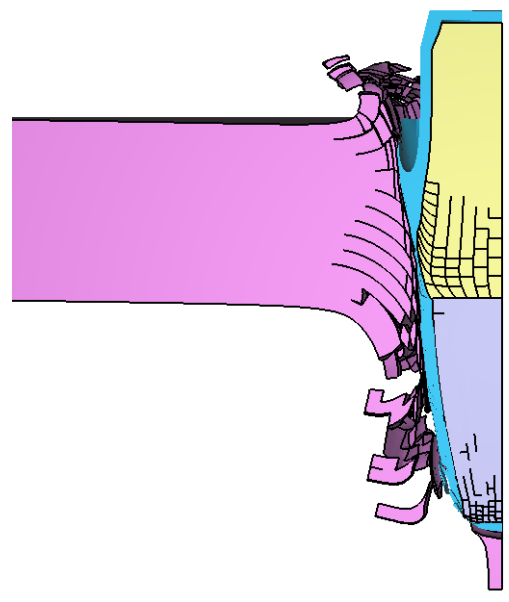

Figure 4.22 - Simulated impact of an M855 bullet with 0.250 " thick aluminum plate, represented with a high density mesh. 


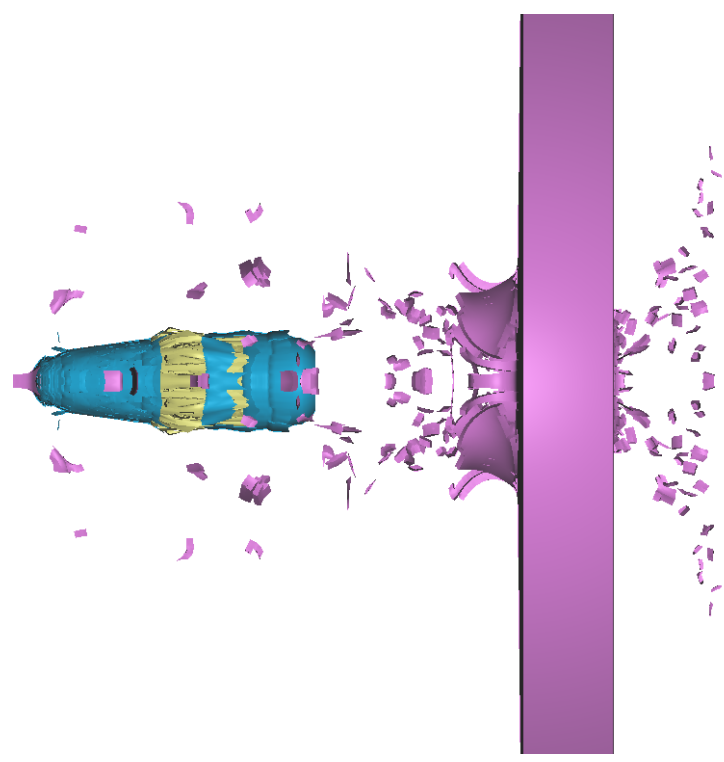

Figure 4.23 - Side view of a simulated impact of an M855 bullet with 0.250 " thick aluminum plate, represented with a high density mesh.

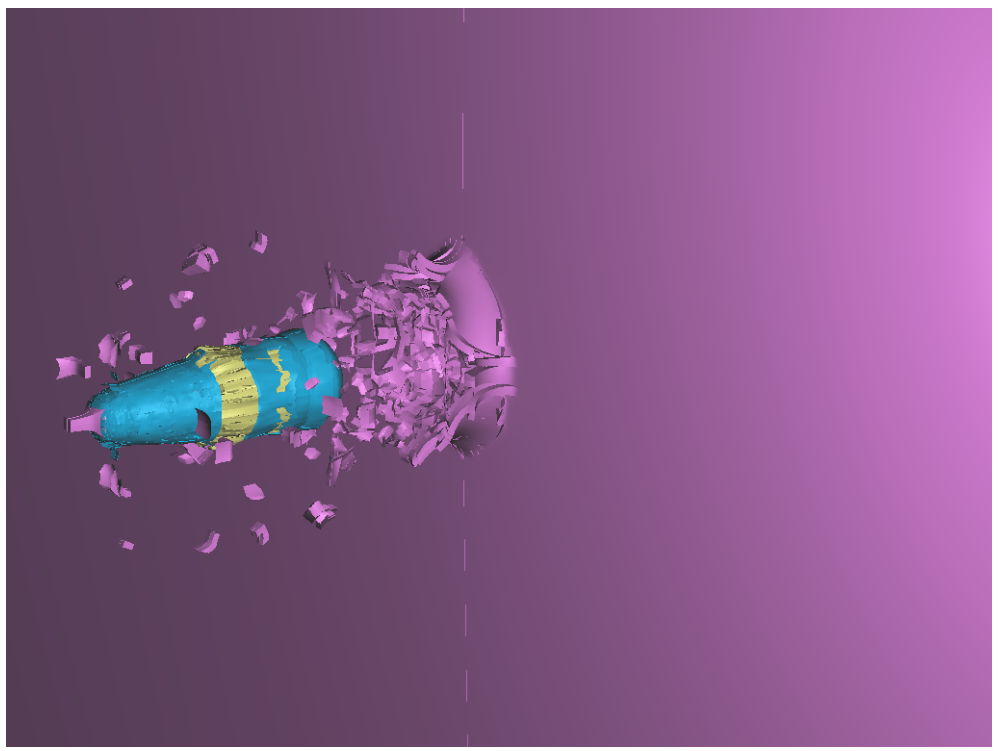

Figure 4.24 - Three quarters view of a simulated impact of an M855 bullet with 0.032" thick aluminum plate, represented with a high density mesh. 


\section{CHAPTER 5}

\section{CONCLUSION}

With advancements in modern combat and weaponry, particularly armor-piercing ammunition, personal body armor has evolved, into more complex metal, ceramic, and composite structures. Soldiers require a personal body armor solution durable enough to withstand multiple high-velocity impacts, and light enough to be worn comfortably with a full load and packed multiple days through rough terrain.

The high-impedance layer, as described in Section 1.2, has been the point of focus for this paper. Through experimentation and simulation a first step towards the evaluation of titanium as an alternative to ceramics has been made. A nominal plate thickness of 0.125 " titanium was observed to cause increased bullet fragmentation (Section 4.1.2), a main goal of the high-impedance layer (Section 1.2). Increased fragmentation was also observed, though to a lesser extent, in simulations discussed in Section 4.3.

Experimental results did not perfectly align with simulated predictions. However, the underlying physics of the model are in general agreement. This suggests that with more advanced instrumentation, the basic model presented here could be tuned to more closely depict experimentally observed results. Future work with high speed video would provide the best comparisons. Generating better information about post impact bullet velocities, as well as information about the orientation of the bullet upon impact. 
The experimental results alone give credence to the idea that titanium could replace ceramics as a high-impedance layer. The simulation and modeling conducted provide a framework for future research, and more rapid design iterations. 


\section{BIBLIOGRAPHY}

[1] Murat Buyuk, Cing-Dao Steve Kan, Nabih E Bedewi, A Durmus, and S Ulku. Moving beyond the finite elements, a comparison between the finite element methods and meshless methods for a ballistic impact simulation. In 8th International LS-DYNA users conference, 2004.

[2] Thomas P Ehrhart. Increasing small arms lethality in afghanistan taking back the infantry half-kilometer. Technical report, DTIC Document, 2009.

[3] AS Fallah, K Micallef, GS Langdon, WC Lee, PT Curtis, and LA Louca. Dynamic response of dyneema $\AA$ hb26 plates to localised blast loading. International Journal of Impact Engineering, 73:91-100, 2014.

[4] DP Goncalves, FCL De Melo, AN Klein, and HA Al-Qureshi. Analysis and investigation of ballistic impact on ceramic/metal composite armour. International Journal of Machine Tools and Manufacture, 44(2):307-316, 2004.

[5] GT Gray, Shuh Rong Chen, W Wright, and MF Lopez. Constitutive equations for annealed metals under compression at high strain rates and high temperatures. Los Alamos National Laboratory, Los Alamos, 1994.

[6] G Kay. Failure modeling of titanium-61-4v and 2024-t3 aluminum with the johnson-cook material model. Technical Rep., Lawrence Livermore National Laboratory, Livermore, CA, 2002.

[7] Hubert W Meyer and David S Kleponis. Modeling the high strain rate behavior of titanium undergoing ballistic impact and penetration. International Journal of Impact Engineering, 26(1):509-521, 2001. 
[8] CW Ong, CW Boey, Robert S Hixson, and Jose O Sinibaldi. Advanced layered personnel armor. International Journal of Impact Engineering, 38(5):369-383, 2011.

[9] Leonard E. Schwer. Preliminary assessment of non-lagrangian methods for penetration simulation. 8th International LS-DYNA Users Conference, 8.

[10] Joseph T South and Larry W Burton. Finite element modeling and analysis of an m855 cartridge. Technical report, DTIC Document, 2004.

[11] Tomasz Wierzbicki. Petalling of plates under explosive and impact loading. International Journal of Impact Engineering, 22(9-10):935 - 954, 1999. 